\title{
APLICAÇÃO DE MODELOS MULTINÍVEIS NA ANÁLISE DE DADOS DE MEDIDAS REPETIDAS NO TEMPO
}

\author{
GENEVILE CARIFE BERGAMO
}

\begin{abstract}
Dissertação apresentada à Escola Superior de Agricultura "Luiz de Queiroz", Universidade de São Paulo, para obtenção do título de Mestre em Agronomia, Área de Concentração: Estatística e Experimentação Agronômica.
\end{abstract}

PIRACICABA

Estado de São Paulo - Brasil

Setembro - 2002 


\title{
APLICAÇÃO DE MODELOS MULTINÍVEIS NA ANÁLISE DE DADOS DE MEDIDAS REPETIDAS NO TEMPO
}

\section{GENEVILE CARIFE BERGAMO}

Engenheiro Agrônomo

\section{Orientador: Prof. Dr. JOSÉ EDUARDO CORRENTE}

\begin{abstract}
Dissertação apresentada à Escola Superior de Agricultura "Luiz de Queiroz", Universidade de São Paulo, para obtenção do título de Mestre em Agronomia, Área de Concentração: Estatística e Experimentação Agronômica.
\end{abstract}

PIRACICABA

Estado de São Paulo - Brasil

Setembro - 2002 
Dados Internacionais de Catalogação na Publicação (CIP) DIVISÃo DE BIBLIOTECA E DOCUMENTAÇÃO - Campus "Luiz de Queiroz"/USP

\author{
Bergamo, Genevile Carife \\ Aplicaçăo de modelos multiníveis na análise de dados de medidas repetidas no \\ tempo / Genevile Carife Bergamo. - - Piracicaba, 2002. \\ 70 p. \\ Dissertaçăo (mestrado) - - Escola Superior de Agricultura Luiz de Queiroz, 2002. \\ Bibliografia. \\ 1. Análise de dados 2. Estatistica aplicada 3. Medidas repetidas 4. Modelos \\ matemáticos I. Título
}

CDD 519.5 


\section{DEDICATÓRIA}

\section{$\grave{A}$ \\ Deus}

Jamais teria conseguido realizar esse trabalho sem a

Sua graça.

Ao meu grande amor,

\section{Maria Inêz Barbosa Braga Bergamo,}

pelo incentivo constante, por suportar a ausência e as privações,

a minha mãe Nair P. C. Bergamo (in memoriam), pela dedicação durante toda sua vida e ao meu pai Casério Bergamo pelo estímulo e apoio.

\footnotetext{
Aos

"meninos", sempre felizes, alegrando minha vida.
} 


\section{AGRADECIMENTOS}

Ao Prof. Dr. José Eduardo Corrente, meu grande amigo e orientador, pelo conhecimento compartilhado, empenho e apoio tornando possível a realização desse trabalho.

Ao Prof. Dr. Aluísio J. D. Barros pelos primeiros passos e a possibilidade de utilização do software MLwiN.

Ao gerente administrativo do ambulatório municipal "Dr. Plínio do Prado Coutinho" Heródoto Landre, por permitir o acesso aos prontuários dos idosos.

À querida enfermeira, responsável pelo Programa de Atenção ao Idoso, Sônia Maria de Souza Lima Paes pelos esclarecimentos sobre o desenvolvimento do "PAI".

À Universidade de Alfenas, na pessoa do Reitor Edson Antônio Velano, do Gestor de Pesquisa e Pós-Graduação João Batista Magalhães e dos Pró-Reitores Fuad Haddad e Hudson Carvalho Bianchini (velho companheiro), por posssibilitar o afastamento das minhas atividades de docência.

À Diretora do Instituto de Farmácia e Nutrição, $\operatorname{Prof}^{a}$. Marlene Leite Godoy V. de Souza, pela amizade e incentivo.

Aos professores e funcionários do Departamento de Ciências Exatas da ESALQ USP, pela atenção e amizade.

Aos colegas de turma, Andréia, Cristiano, Denise, Flávia, Glaucy, Janaína, Mariana, Osmir pela amizade e companheirismo, além dos "importados" Ezequiel e Ramiro pela companhia durante as refeições. 
Aos amigos do doutorado, Adriano, Andre, Antônio Carlos, "Cris", Maurício, Renato, Sueli, Telde, pelo incentivo e colaboração, em especial ao Silvano pelo material e "dicas" dos softwares.

Às funcionárias do "RUCAS" sempre pacientes e solícitas, em especial à $D^{n a}$ Expedita pelas "frutas".

Às pessoas que compartilharam direta ou indiretamente para realização desse trabalho. 


\section{SUMÁRIO}

\section{Página}

LISTA DE FIGURAS

viii

LISTA DE TABELAS ix

RESUMO - xii

SUMMARY Xiv

1 INTRODUÇÃO

2 REVISÃO DE LITERATURA 4

2.1 Modelos multiníveis . . . . . . . . . . . . . . . . . . 4

2.2 Estrutura geral dos modelos multiníveis f . . . . . . . . . . . 6

2.2.1 O modelo de regressão linear simples . . . . . . . . . . . . . . . . 6

$2.2 .2 \quad$ O modelo multinível com dois níveis f . . . . . . . . . . . . . 7

2.3 Estimação . . . . . . . . . . . . . . . . . . . . . . . . . . . . . . 14

2.4 Testes de Hipóteses . . . . . . . . . . . . . . . . . . . . . . . . . . . . . 16

2.4.1 Teste de Hipótese para os efeitos fixos $\gamma \ldots \ldots$. . . . . . . . 16

2.4.2 Teste de Hipótese para os componentes de variância $R$ e $G \ldots \ldots$

2.5 Análise de dados de medidas repetidas . . . . . . . . . . . . . . . 17

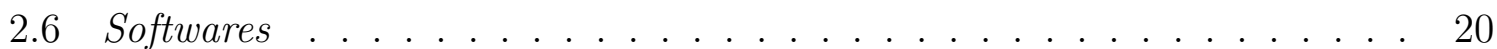

2.6.1 Programa MLwiN . . . . . . . . . . . . . . . . . . . . 21

2.6 .2 PROC MIXED do SAS . . . . . . . . . . . . . . . 22 
3 METODOLOGIA 23

3.1 Análise dos dados do Programa de Atenção ao Idoso - "PAI" . . . . . . . 23

3.1.1 Descrição do experimento . . . . . . . . . . . . . . . . 23

3.1.2 Descrição dos modelos . . . . . . . . . . . . . . . . . 25

3.2 Análise dos dados do teor de proteína no leite . . . . . . . . . . . 28

3.2.1 Descrição do experimento . . . . . . . . . . . . . . . . . . . . . . 28

3.2.2 Descrição dos modelos . . . . . . . . . . . . . . . . . . . . . . . 28

4 RESULTADOS E DISCUSSÃO 30

4.1 Resultados da análise dos dados do "PAI" . . . . . . . . . . . . . . . . . 30

4.2 Resultados da análise dos dados do teor de proteína no leite . . . . . . . 50

5 CONCLUSÕES $\quad 57$

REFERÊNCIAS BIBLIOGRÁFICAS $\quad 59$

$\begin{array}{lc}\text { APÊNDICE } & 62\end{array}$ 


\section{LISTA DE FIGURAS}

\section{Página}

1 Estrutura dos dados para um modelo hierárquico em três níveis, com medidas repetidas no tempo . . . . . . . . . . . . . . . 2

2 Estrutura dos dados para um modelo hierárquico em três níveis . . . . . 4

3 Estrutura dos dados para um modelo hierárquico em dois níveis . . . . . 5

4 Estrutura dos dados para um modelo multinível com dois níveis . . . . . 8

5 Gráfico do modelo final para a variável $\operatorname{pass}_{i j} \ldots \ldots$. . . . . . . . . 35

6 Gráfico do modelo final para a variável pads $\mathrm{paj}_{i j} \ldots \ldots$. . . . . . . . . 41

$7 \quad$ Gráfico do modelo final para a variável $\operatorname{pasp}_{i j} \ldots \ldots$. . . . . . . . . 46

8 Gráfico do modelo final para a variável $\operatorname{padp}_{i j} \ldots \ldots$. . . . . . . . . . 49

9 Gráfico do modelo final para a variável teor ${ }_{i j} \ldots \ldots$. . . . . . . . . 56 


\section{LISTA DE TABELAS}

Página

1 Estrutura básica dos dados para estudos longitudinais . . . . . . . . . . . 19

2 Estimativa dos parâmetros do modelo (16) considerando apenas os interceptos para os dados de pressão arterial sistólica sentado - "PAI" . . . . . 30

3 Estimativa dos parâmetros do modelo (17) considerando IMCC como uma variável explanatória para os dados de pressão arterial sistólica sentado "PAI" ........................... . . . . . . . .

4 Estimativa dos parâmetros do modelo (18) considerando o IMCC com efeito aleatório para o nível dos idosos para os dados de pressão arterial sistólica sentado - "PAI" . . . . . . . . . . . . . . . . . . 33

5 Estimativa dos parâmetros do modelo (19) considerando o IMCC com efeito aleatório para o nível dos idosos mais as variáveis explanatórias desse nível para os dados de pressão arterial sistólica sentado - "PAI" . . 34

6 Estimativa dos parâmetros do modelo (16) considerando apenas os interceptos para os dados de pressão arterial diastólica sentado - "PAI"

7 Estimativa dos parâmetros do modelo (17) considerando IMCC como uma variável explanatória para os dados de pressão arterial diastólica sentado

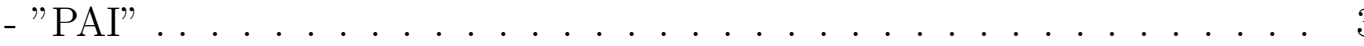

8 Estimativa dos parâmetros do modelo (18) considerando o IMCC com efeito aleatório para o nível dos idosos para os dados de pressão arterial diastólica sentado - "PAI" . . . . . . . . . . . . . . . 38 
9 Estimativa dos parâmetros do modelo (19) considerando o IMCC com efeito aleatório para o nível dos idosos mais as variáveis explanatórias desse nível para os dados de pressão arterial diastólica sentado - "PAI" .

10 Estimativa dos parâmetros do modelo (16) considerando apenas os interceptos para os dados de pressão arterial sistólica em pé - "PAI" . . . . . 41

11 Estimativa dos parâmetros do modelo (17) considerando IMCC como uma variável explanatória para os dados de pressão arterial sistólica em pé "PAI" . . . . . . . . . . . . . . . . . . .

12 Estimativa dos parâmetros do modelo (18) considerando o IMCC com efeito aleatório para o nível dos idosos para os dados de pressão arterial sistólica em pé - "PAI" . . . . . . . . . . . . . . . . . .

13 Estimativa dos parâmetros do modelo (19) considerando o IMCC com efeito aleatório para o nível dos idosos mais as variáveis explanatórias desse nível para os dados de pressão arterial sistólica em pé - "PAI" . . .

14 Estimativa dos parâmetros do modelo (16) considerando apenas os interceptos para os dados de pressão arterial diastólica em pé - "PAI" . . . . . 46

15 Estimativa dos parâmetros do modelo (17) considerando IMCC como uma variável explanatória para os dados de pressão arterial diastólica em pé "PAI" . . . . . . . . . . . . . . . . . . .

16 Estimativa dos parâmetros do modelo (18) considerando o IMCC como efeito aleatório para o nível dos idosos para os dados de pressão arterial diastólica em pé - "PAI" . . . . . . . . . . . . . . . . .

17 Estimativa dos parâmetros do modelo análogo ao (16) para os dados de teor de proteína do leite, considerando apenas os interceptos. . . . . . . .

18 Estimativa dos parâmetros do modelo análogo ao (17) considerando TEMPO como uma variável explanatória para os dados de teor de proteína do

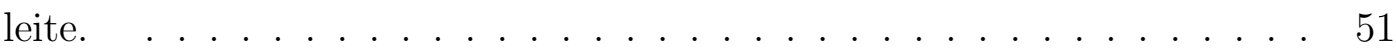


19 Estimativa dos parâmetros do modelo análogo ao (18) considerando o TEMPO com efeito aleatório para o nível dos animais para os dados de teor de proteína no leite. . . . . . . . . . . . . . . . 53

20 Estimativa dos parâmetros do modelo (20) considerando o TEMPO com efeito aleatório para o nível dos animais e a variável explanatória dieta para os dados de teor de proteína no leite. . . . . . . . . . . . . . . . . 54

21 Estimativa dos parâmetros do modelo (21) considerando o TEMPO com efeito aleatório para o nível dos animais e suas interações com a variável explanatória desse nível para os dados de teor de proteína no leite. . . . 55 


\title{
APLICAÇÃO DE MODELOS MULTINÍVEIS NA ANÁLISE DE DADOS DE MEDIDAS REPETIDAS NO TEMPO
}

\author{
Autor: GENEVILE CARIFE BERGAMO \\ Orientador: Prof. Dr. JOSÉ EDUARDO CORRENTE
}

\section{RESUMO}

Em muitos trabalhos científicos, é comum encontrar os dados estruturados de forma hierárquica, ou seja, os indivíduos em estudo estão agrupados em unidades de nível mais baixo, que por sua vez pertencem a unidades de um nível mais alto e assim sucessivamente. Na análise desse tipo de dados é importante levar em conta a estrutura hierárquica uma vez que, não fazê-la, pode implicar na superestimação dos coeficientes do modelo em estudo. Assim, para facilitar a análise de dados seguindo uma estrutura hierárquica, foram desenvolvidos os modelos multiníveis. Tais modelos levam em conta toda a variabilidade existente para os dados num mesmo nível como nos diferentes níveis da hierarquia. No caso da análise de dados de medidas repetidas no tempo, uma estrutura hierárquica em dois níveis pode ser considerada, organizando as ocasiões de medidas, no primeiro nível, para cada indivíduo no segundo nível. Neste trabalho, é feita uma abordagem dos modelos multiníveis para vários níveis da hierarquia bem como os métodos de estimação e 
teste dos parâmetros envolvidos no modelo. Como aplicação, foram analisados dados provenientes do Programa de Atenção ao Idoso (PAI), desenvolvido no ambulatório municipal Dr. Plínio do Prado Coutinho em Alfenas, M.G., em que foram observadas as variáveis Índice de Massa Corpórea (IMC) e Pressão Arterial dos idosos durante 22 meses. Também, foram analisados dados referentes ao teor de proteína no leite de 79 vacas australianas, coletados durante 19 semanas após o parto e submetidas a três dietas (Diggle et al., 1994). Para os dados do "PAI", foi possível verificar que as diferentes medidas de pressão arterial estão relacionadas positivamente com o IMC ao longo do tempo, independente de sexo, idade e estado civil. Já nos dados relativos ao teor de proteína no leite, notou-se uma redução do teor de proteína no leite ao longo do tempo, independente dos tratamentos aplicados. Foram utilizados os softwares MLwiN e SAS para a realização das análises. 


\section{MULTILEVEL MODELS APPLIED IN THE ANALYSIS OF REPEATED MEASURE DATA}

Author: GENEVILE CARIFE BERGAMO Adviser: Prof. Dr. JOSÉ EDUARDO CORRENTE

\section{SUMMARY}

It is common to find data structured in a hierarchical form in several scientific works, that is, the studied subjects are nested in the lowest level unites, that belong to the highest level unites, and so on. To analyze these sort of data it is important to take in account the hierarchical structure once, if does not do it, the coefficients can be overestimated in the studied model. Then, in order to become easier the data analysis according to the hierarchical structure, multilevel models were developed. Such models take into account all the existing variability for the data at the same level as well as in different levels of the hierarchy. In the case of repeated measure data, a two levels hierarchical structure can be considered, organizing the occasions at the first level for each subject at the second level. In this work, na approach of the multilevel models for several levels are made as well as the estimation methods and the tests for the involved parameters in the model. As an 
application, data from the Elderly Care Program (ECP), developed at outpatient clinic Dr. Plinio do Prado Coutinho at Alfenas, M.G., where the Body Mass Index and the Bloody Pressure were observed from elderly people for 22 months. Also, it was analyzed the milk protein content of 79 australian cows during 19 weeks after calving and subject to three diets (Diggle et al., 1994). For the data of the ECP it was possible to observe that the bloody pressure are positively related to the occasions, independently of sex, race and marital status. For the data form the milk protein content, a reduce in the content in the occasions even after the diets are included. MLwiN and SAS softwares were used to run the analysis. 


\section{INTRODUÇÃO}

Em estudos biológicos bem como em outras áreas do conhecimento, é comum encontrar os dados estruturados de forma hierárquica, ou seja, os indivíduos em estudo estão agrupados em unidades de nível mais baixo, que por sua vez pertencem a unidades de um nível mais alto e assim sucessivamente.

Reconhecer e levar em conta a estrutura hierárquica dos dados é importante, uma vez, que não fazê-la pode implicar na superestimação dos coeficientes do modelo em estudo.

Em modelos de regressão tradicionais, existe a suposição de independência entre os indivíduos. No entanto, quando os dados são estruturados em hierarquias, as unidades de um mesmo nível pertencentes a uma unidade de nível mais alto, raramente são independentes. Isso acontece porque essas unidades compartilham de um mesmo ambiente ou apresentam características semelhantes. Assim, a suposição de independência foi violada e passa a existir correlação entre essas unidades. É preciso que o modelo a ser ajustado leve em conta tais correlações. Para isto, foram desenvolvidos modelos multiníveis, os quais contemplam uma estrutura hierárquica dos dados, considerando todas as correlações existentes entre as observações nos diferentes níveis de hierarquia.

Nos modelos multiníveis, as unidades de cada nível são vistas como tendo um efeito aleatório, ou seja, são amostras aleatórias de uma população dessas unidades. Estes efeitos aleatórios são responsáveis no modelo por coeficientes aleatórios que levam em conta a variabilidade entre essas unidades, seja de forma simples, através da variabilidade nos interceptos, ou de forma mais complexa, 
através da variabilidade nas inclinações. Além disso, os modelos multiníveis com efeitos aleatórios possuem a vantagem em relação aos tradicionais de acomodarem a estrutura hierárquica aos dados.

Um exemplo da estrutura hierárquica pode ser visto na Figura 1. Notase neste caso, que as ocasiões de medidas, representando o nível um, são feitas para cada indivíduo, do nível dois, em cada localidade, do nível 3. Assim, para este caso, tem-se um experimento de medidas repetidas no tempo colocado sob a forma de uma estrutura hierárquica.

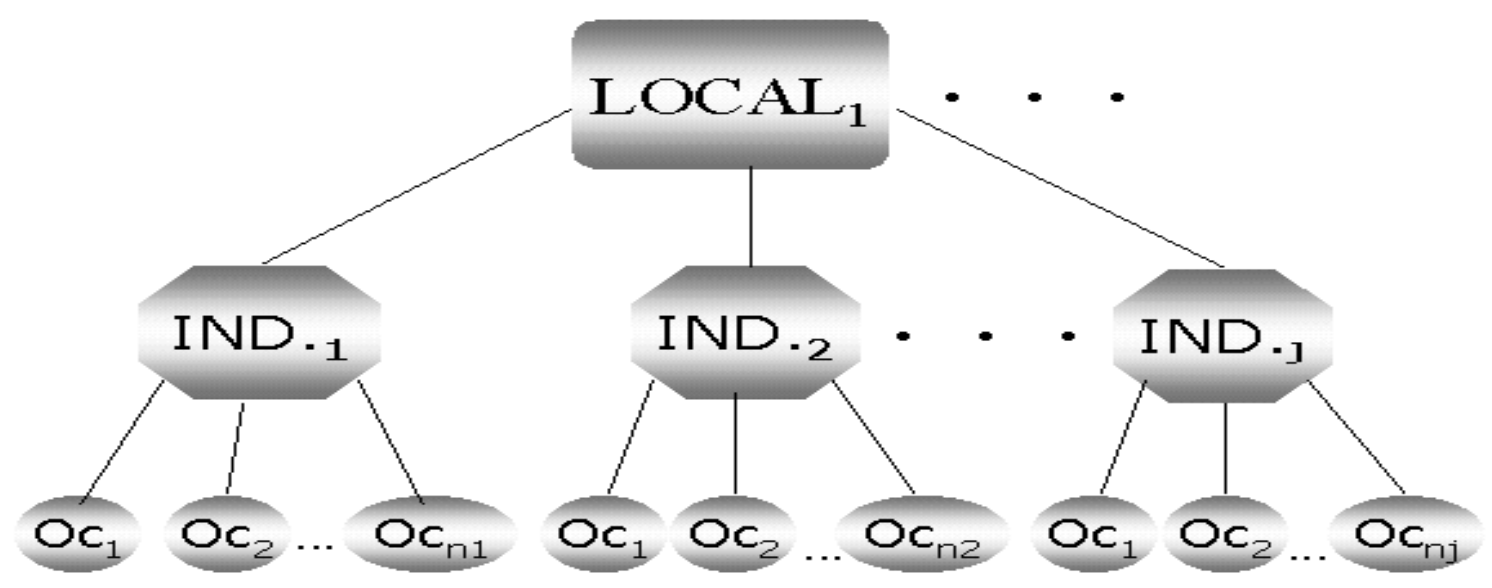

Figura 1 - Estrutura dos dados para um modelo hierárquico em três níveis, com medidas repetidas no tempo

Quando os dados estão dispostos segundo uma estrutura hierárquica, pode existir uma correlação entre os níveis da hierarquia, sendo que através de uma análise simples, essa correlação, pode não ser detectada. Diante deste fato, o objetivo deste trabalho consiste no estudo dos modelos multiníveis de um modo geral, considerando os problemas decorrentes dessa estrutura hierárquica e aplicação de 
tais modelos na análise de dados de medidas repetidas no tempo, em dois conjuntos de dados: o primeiro referente ao "PAI" (Programa de Atenção ao idoso) do Ambulatório Dr. Plínio do Prado Coutinho em Alfenas, M.G., e o segundo, tirado do livro de Diggle et al. (1994), sobre a análise do teor de proteína no leite em vacas australianas, submetidas a três diferentes dietas. 


\section{REVISÃO DE LITERATURA}

\subsection{Modelos multiníveis}

Modelos multiníveis são modelos que levam em conta a estrutura hierárquica para as variáveis em estudo, isto é, unidades agrupadas em um nível mais baixo, as quais estão agrupadas dentro de outras unidades de um nível mais alto e assim sucessivamente. Como exemplo, a Figura 2 mostra uma estrutura hierárquica, considerando um estudo em que o interesse está na avaliação das escolas através do desempenho de seus alunos e professores. Assim, os alunos estão agrupados por professores e esses agrupados por escolas.

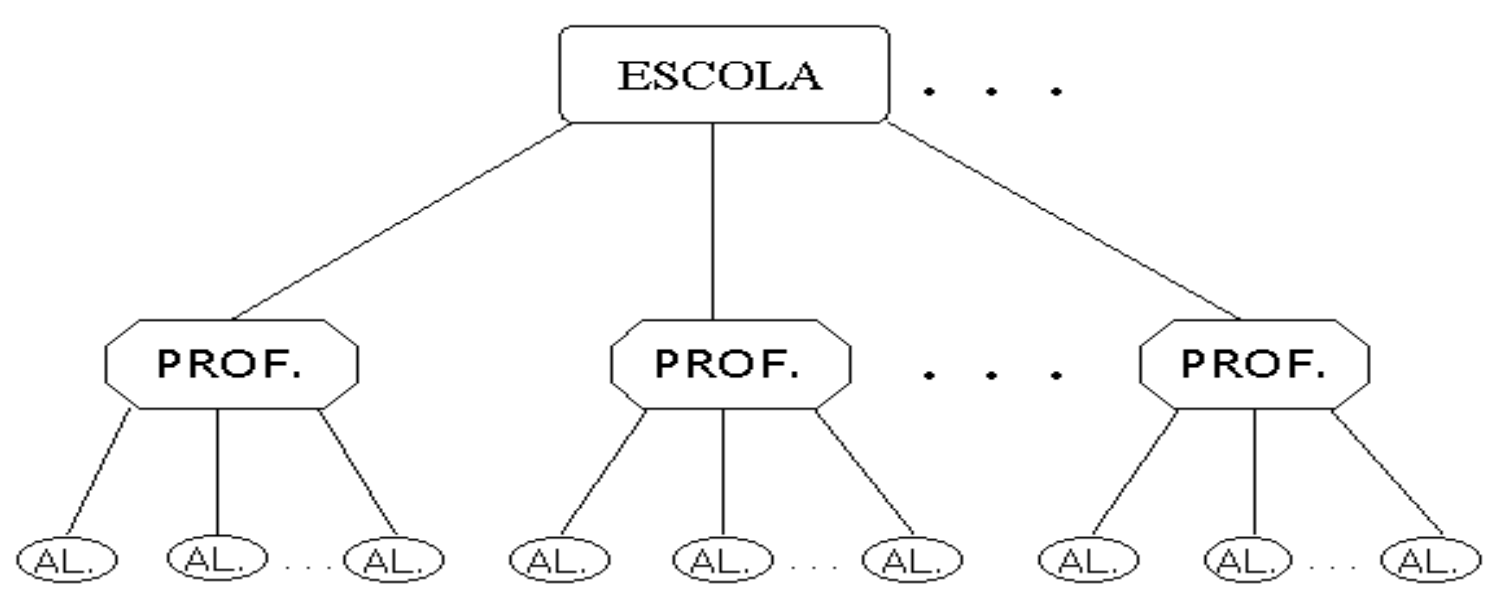

Figura 2 - Estrutura dos dados para um modelo hierárquico em três níveis 
Já a Figura 3 mostra uma estrutura hierárquica com medidas repetidas no tempo em que, as ocasiões de medida estão no primeiro nível da estrutura hierárquica. Portanto, qualquer experimento com medidas repetidas no mesmo indivíduo ao longo do tempo, pode ser analisado segundo essa estrutura. Como exemplo, os experimentos para verificação de possíveis fatores que afetam o crescimento das plantas, em que as ocasiões de medida (primeiro nível) são agrupadas às plantas (segundo nível).

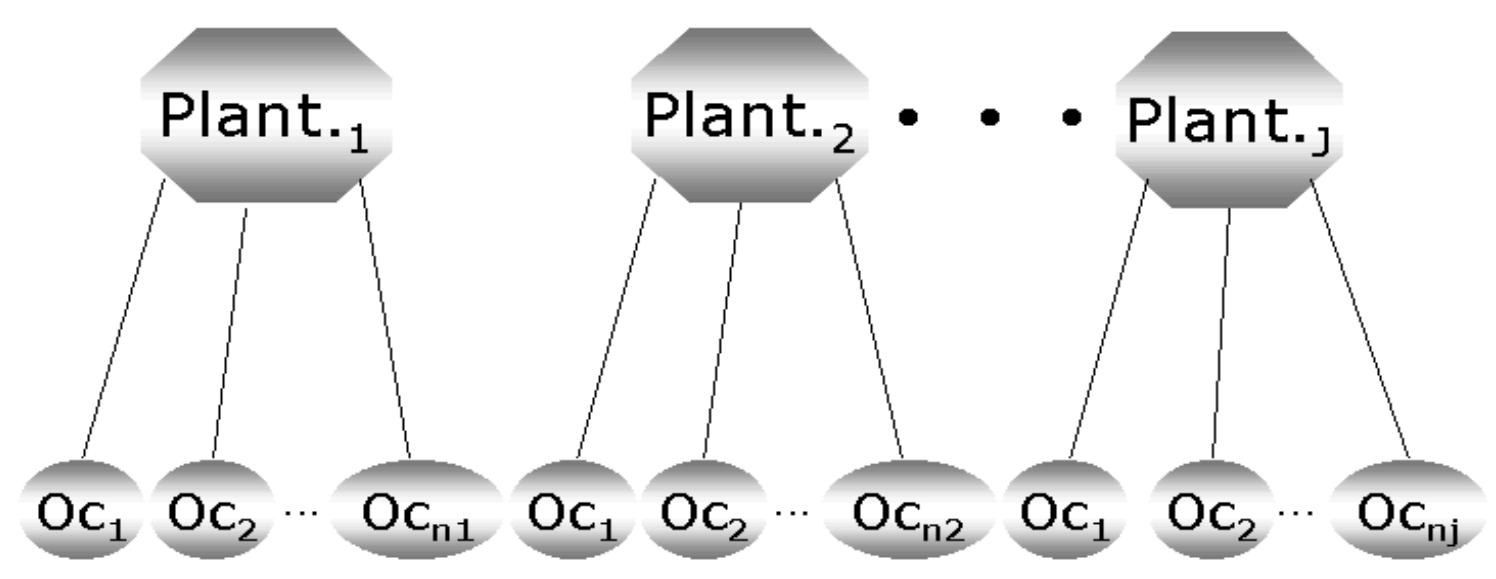

Figura 3 - Estrutura dos dados para um modelo hierárquico em dois níveis

A utilização de modelos multiníveis está relacionada a um estudo com crianças do ensino fundamental conduzido nos anos 70 na Inglaterra. Bennett (1976) alegou que as crianças expostas à uma maneira formal de ensinar a ler, exibiam um aprendizado maior do que as não expostas. Os dados foram analisados utilizando técnicas de regressão múltipla tradicionais, considerando as crianças individualmente como sendo as unidades de análise e ignorando os agrupamentos para professores e classes. Os resultados obtidos foram estatisticamente significantes. Subseqüentemente, Aitkin et al. (1981) demonstraram que, ao considerar a análise com as crianças agrupadas em classes, as diferenças significantes desapareciam, e as que se submeteram ao ensino formal, não apresentaram qualquer diferença em relação às 
demais (Goldstein, 1995).

Aitkin \& Longford (1986) iniciaram, assim, uma série de procedimentos, resultando nas idéias centrais dos modelos multiníveis. Bryk \& Raudenbush (1992) discutiram modelos lineares multiníveis com 2 e 3 níveis para aplicações específicas a dados educacionais e experimentos com medidas repetidas. Longford (1993) fornece uma orientação mais teórica dos modelos multiníveis incluindo a discussão de um modelo multinível para análise fatorial, modelos com respostas categorizadas e outros modelos multivariados. Atualmente, o desenvolvimento desses modelos está voltado para as áreas de dados com respostas discretas, modelos de séries temporais, classificações cruzadas, dados perdidos e modelos não lineares, com aplicação na educação, epidemiologia, geografia, crescimento animal e vegetal, pesquisas domiciliares, dentre outras (Goldstein, 1995).

\subsection{Estrutura geral dos modelos multiníveis}

\subsubsection{O modelo de regressão linear simples}

De acordo com Neter et al. (1990), no modelo de regressão linear simples, a relação entre a variável resposta e a variável explanatória, geralmente, é escrita como:

$$
Y_{i}=\beta_{0}+\beta_{1} X_{i}+\epsilon_{i} \quad \operatorname{com} i=1, \ldots, n
$$

em que

$Y_{i}$ representa a resposta do $i$-ésimo indivíduo;

$\beta_{0}$ o valor esperado da variável resposta $Y_{i}$ para $X_{i}$ igual a zero;

$\beta_{1}$ a mudança esperada em $Y_{i}$ quando $X_{i}$ aumenta de uma unidade; 
$X_{i}$ é variável explanatória do $i$-ésimo indivíduo;

$\epsilon_{i}$ é o erro associado ao $i$-ésimo indivíduo, com as suposições:

$\epsilon_{i} \sim N\left(0, \sigma^{2}\right)$ e $\epsilon_{i}{ }^{\prime}$ s independentes.

Ao se considerar várias retas de regressão, que podem ser originadas de experimentos realizados sob as mesmas condições, pode-se ter um conjunto de retas para explicar a variabilidade do experimento. Assim, comparando-se dois experimentos, o que possui maior intercepto é o que proporciona um melhor resultado na origem. O que possui menor inclinação, mostra uma relação com a variável explanatória mais fraca, o que o torna menos preditivo em relação ao outro experimento. Desse modo, se o objetivo é estudar não apenas um determinado evento, mas estudar tudo o que os envolve, é possível ajustar um modelo que leve em conta toda a variabilidade entre os experimentos e incorpore os diferentes aspectos de cada um deles. Uma maneira de se levar em conta tal tipo de análise, é considerar os dados de acordo com uma estrutura hierárquica.

\subsubsection{O modelo multinível com dois níveis}

Em modelos hierárquicos ou modelos multiníveis com dois níveis, as observações são classificadas como unidades de nível 1 e unidades de nível 2, ou seja, ocorrem $n_{j}$ unidades do nível 1 para cada unidade $j(j=1,2, \ldots, J)$ do nível 2. Neste caso, os dados não precisam ser balanceados, isto é, não é necessário que $n_{j}=n_{k}$ para $j \neq k$. A Figura 4 mostra os dados estruturados segundo um modelo multinível com dois níveis. 


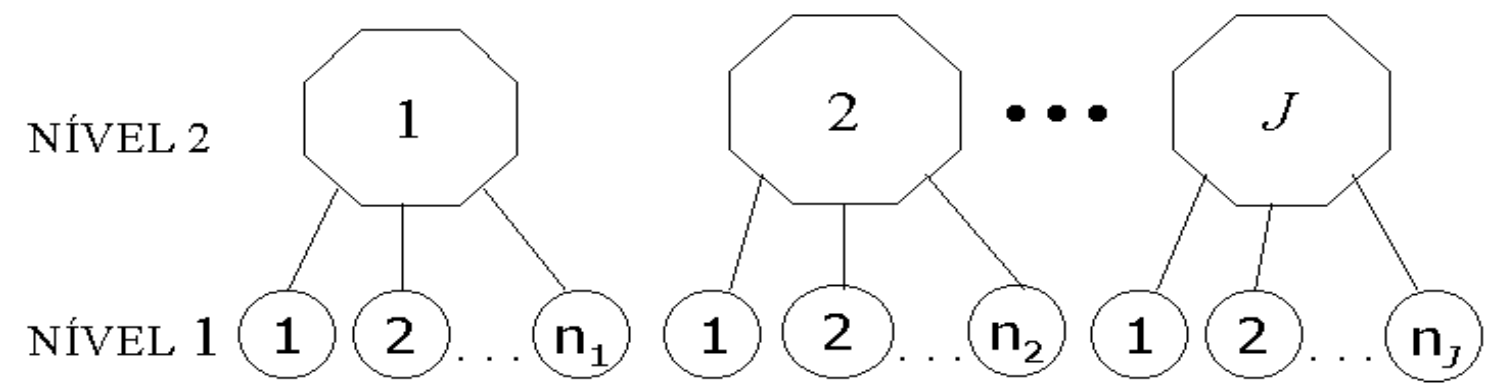

Figura 4 - Estrutura dos dados para um modelo multinível com dois níveis

Assim, os modelos para o nível 1 são desenvolvidos separadamente em cada unidade $j$ do nível 2, levando-se em consideração uma possível variação de interceptos e inclinações. Considerando o caso de uma variável resposta $Y$ e uma única variável explanatória $X$ do nível 1, o modelo do nível 1 é da forma:

$$
Y_{i j}=\beta_{0 j}+\beta_{1 j} X_{i j}^{*}+\epsilon_{i j}
$$

com $i=1,2, \ldots, n_{j}$ e $j=1,2, \ldots, J$ em que

$Y_{i j}$ é a variável resposta do $i$-ésimo indivíduo do nível 1 para o $j$-ésimo nível 2,

$X_{i j}^{*}$ a variável explanatória em sua medida original centrada na média geral $\bar{X}_{\text {.. ou }}$ centrada na média de uma unidade do nível $2 \bar{X}_{. j}$, medidas na $i$-ésima unidade do nível 1 agrupadas para a $j$-ésima unidade do nível 2;

$\beta_{0 j}$ é o intercepto para a $j$-ésima unidade do nível 2 ;

$\beta_{1 j}$ é a inclinação associada à variável explanatória $X_{i j}^{*}$ da $i$-ésima unidade do nível 1 para a $j$-ésima unidade do nível 2 ;

$\epsilon_{i j}$ é o erro aleatório associado à $i$-ésima unidade do nível 1 agrupado para a $j$ ésima unidade do nível 2, com as suposições:

$$
\epsilon_{i j} \sim N\left(0, \sigma^{2}\right) \text { e } \epsilon_{i j} \text { 's independentes. }
$$


A interpretação dos parâmetros do modelo (particularmente os interceptos $\beta_{0 j}$ para todo $j$ ) depende do modo como as variáveis explanatórias do nível 1 são consideradas. Pode-se dizer que:

- se a variável explanatória do nível $1, X_{i j}^{*}$, é considerada em sua medida original, isto é, como $X_{i j}$, então o intercepto $\beta_{0 j}$ é o valor esperado da variável resposta $Y_{i j}$ quando $X_{i j}$ for igual a zero;

- agora, se a variável explanatória do nível $1, X_{i j}^{*}$, está centrada na sua média geral, isto é, centrada em $\bar{X}_{. .}$, então, o intercepto $\beta_{0 j}$, é interpretado como a média da $j$-ésima unidade do nível 2 ajustada para a variável $X$;

- mas, se a variável explanatória $X_{i j}^{*}$, do nível 1 , é centrada em $\bar{X}_{. j}$, ou seja, na respectiva média da unidade do nível 2 , então o intercepto $\beta_{0 j}$ é interpretado como a média não ajustada da variável resposta $Y_{i j}$;

- finalmente, se a variável explanatória do nível $1, X_{i j}^{*}$ é centrada em qualquer outro valor médio, então, o intercepto $\beta_{0 j}$ é interpretado como o valor esperado da variável resposta $Y_{i j}$ para cada valor da variável explanatória.

No modelo para o nível 2, considerando as variáveis respostas $Y_{i j}$ e a variável explanatória $X_{i j}^{*}$, têm-se $J$ modelos iguais aos mostrados em (1) em que, para cada modelo há interceptos $\beta_{0 j}$ 's e inclinações $\beta_{1 j}$ 's diferentes, para $j=1, \ldots, J$. Para os modelos no nível 2, estes coeficientes de regressão são considerados como variáveis resposta, isto é:

$$
\begin{aligned}
& \beta_{0 j}=\gamma_{00}+\nu_{0 j} \\
& \beta_{1 j}=\gamma_{10}+\nu_{1 j},
\end{aligned}
$$

em que

$\gamma_{00}$ é o valor esperado dos interceptos nas unidades do nível 2;

$\gamma_{10}$ é o valor esperado dos interceptos das inclinações na população de unidades do nível 2 ; 
$\nu_{0 j}$ é o efeito aleatório da $j$-ésima unidade do nível 2 no intercepto $\beta_{0 j}$;

$\nu_{1 j}$ é o efeito aleatório da $j$-ésima unidade do nível 2 na inclinação $\beta_{1 j}$;

$\tau_{00}$ é a variância populacional dos interceptos;

$\tau_{11}$ a variância populacional das inclinações;

$\tau_{01}$ a covariância entre $\beta_{0 j}$ e $\beta_{1 j}$, com as suposições:

$$
\begin{aligned}
& \nu_{0 j} \sim N\left(0, \tau_{00}\right) \text { e } \nu_{0 j} \text { 's independentes; } \\
& \nu_{1 j} \sim N\left(0, \tau_{11}\right) \text { e } \nu_{1 j} \text { 's independentes; } \\
& \operatorname{Cov}\left(\nu_{0 j}, \nu_{1 j}\right)=\tau_{01} \\
& \nu_{0 j} \text { 's e } \nu_{1 j} \text { 's independentes dos } \epsilon_{i} \text { 's. }
\end{aligned}
$$

Em resumo,

$$
\beta_{0 j} \sim N\left(\gamma_{00}, \tau_{00}\right), \quad \beta_{1 j} \sim N\left(\gamma_{10}, \tau_{11}\right) \quad \text { e } \operatorname{Cov}\left(\beta_{0 j}, \beta_{1 j}\right)=\tau_{01}
$$

Nota-se que, com as equações (2), as unidades do nível 2 não apresentam o mesmo intercepto e a mesma inclinação e os seus efeitos aleatórios, $\nu_{0 j}$ e $\nu_{1 j}$, ajudam a esclarecer estas possíveis diferenças.

Além disso, é possível incluir variáveis explanatórias relacionadas ao nível 2 para explicar a variabilidade entre as unidades do nível 2. Assim, considere as variáveis $W_{j}$ para o nível 2. Os modelos agora para este nível serão da forma:

$$
\begin{aligned}
& \beta_{0 j}=\gamma_{00}+\gamma_{01} W_{j}+\nu_{0 j} \\
& \beta_{1 j}=\gamma_{10}+\gamma_{11} W_{j}+\nu_{1 j},
\end{aligned}
$$

em que

$\beta_{0 j}$ é o intercepto para a $j$-ésima unidade do nível 2 ;

$\beta_{1 j}$ é a inclinação para a $j$-ésima unidade do nível 2 ;

$\gamma_{00}$ é o valor esperado do intercepto para $W_{j}$; 
$\gamma_{10}$ é é o valor esperado das inclinações para $W_{j}$;

$\gamma_{01}$ é o coeficiente de regressão associado à variável explanatória $W_{j}$ do nível 2 relativo ao intercepto;

$\gamma_{11}$ é o coeficiente de regressão associado à variável explanatória $W_{j}$ do nível 2 relativo à inclinação;

$\nu_{0 j}$ é o efeito aleatório da $j$-ésima unidade do nível 2 sobre o intercepto para $W_{j}$;

$\nu_{1 j}$ é o efeito aleatório da $j$-ésima unidade do nível 2 sobre a inclinação para $W_{j}$;

$\tau_{00}$ é a variância populacional dos interceptos corrigida pela variável $W_{j}$;

$\tau_{11}$ a variância populacional das inclinações corrigida pela variável $W_{j}$;

$\tau_{01}$ é a a covariância entre $\beta_{0 j}$ e $\beta_{1 j}$, com as suposições:

$$
\begin{aligned}
& \nu_{0 j} \sim N\left(0, \tau_{00}\right) \text { e } \nu_{0 j} \text { 's independentes; } \\
& \nu_{1 j} \sim N\left(0, \tau_{11}\right) \text { e } \nu_{1 j} \text { 's independentes; } \\
& \operatorname{Cov}\left(\nu_{0 j}, \nu_{1 j}\right)=\tau_{01} ; \\
& \nu_{0 j} \text { 's e } \nu_{1 j} \text { 's independentes dos } \epsilon_{i} \text { 's. }
\end{aligned}
$$

Note que, da mesma forma vista anteriormente para a variável explanatória no nível 1 para o modelo dado em (1), pode-se considerar $W_{j}$ em sua escala original ou uma variável $W_{j}^{*}$ centrada na média geral $\bar{W}_{\text {.. }}$. Incluindo a variável $W_{j}, \tau_{00}, \tau_{11}$ e $\tau_{01}$ são agora componentes de variância e covariância condicionais ou residuais.

Substituindo (3) na equação (1), tem-se:

$$
Y_{i j}=\gamma_{00}+\gamma_{01} W_{j}^{*}+\gamma_{10} W_{j}^{*} X_{i j}^{*}++\gamma_{11} W_{j}^{*} X_{i j}^{*}+\nu_{0 j}+\nu_{1 j} X_{i j}^{*}+\epsilon_{i j}
$$

O modelo combinado (4) envolve as variáveis explanatórias $X_{i j}^{*}$ do nível 1 e $W_{j}^{*}$ do nível 2 , um termo entre os níveis $W_{j}^{*} X_{i j}^{*}$ e um termo complexo 
$\nu_{0 j}+\nu_{1 j} X_{i j}^{*}+\epsilon_{i j}$, considerado como um erro complexo. Ainda tem-se que os erros não são independentes nas unidades de nível 1, ou seja, há uma dependência entre as unidades do nível 1 agrupadas dentro de cada unidade do nível 2 em termos de $\nu_{0 j}$ e $\nu_{1 j}$. Além disso, as variâncias dos erros podem não ser homogêneas se $\nu_{0 j}$ e $\nu_{1 j}$ assumirem diferentes valores dentro de cada unidade do nível 2.

Usando a notação matricial, estes modelos podem ser representados como segue:

- modelo para o nível 1 como em (1):

$$
Y_{j}=X_{j} \beta_{j}+\epsilon_{j}, \quad j=1,2, \ldots, J
$$

em que:

$$
Y_{j}=\left[\begin{array}{c}
Y_{1 j} \\
Y_{2 j} \\
\ldots \\
Y_{n_{j} j}
\end{array}\right], \quad X_{j}=\left[\begin{array}{cc}
1 & X_{1 j} \\
1 & X_{2 j} \\
\ldots & \ldots \\
1 & X_{n_{j j}}
\end{array}\right], \quad \beta_{j}=\left[\begin{array}{c}
\beta_{0 j} \\
\beta_{1 j}
\end{array}\right], \quad \epsilon_{j}=\left[\begin{array}{c}
\epsilon_{1 j} \\
\epsilon_{2 j} \\
\ldots \\
\epsilon_{n_{j} j}
\end{array}\right] .
$$

- modelo para o nível 2 como em (4):

$$
\beta_{j}=W_{j} \gamma+\nu_{j}, \quad j=1,2, \ldots, J
$$

em que:

$$
\beta_{j}=\left[\begin{array}{c}
\beta_{0 j} \\
\beta_{1 j}
\end{array}\right], \quad W_{j}=\left[\begin{array}{cccc}
1 & W_{j} & 0 & 0 \\
0 & 0 & 1 & W_{j}
\end{array}\right], \quad \gamma=\left[\begin{array}{c}
\gamma_{00} \\
\gamma_{01} \\
\gamma_{10} \\
\gamma_{11}
\end{array}\right], \quad \nu_{j}=\left[\begin{array}{c}
\nu_{0 j} \\
\nu_{1 j}
\end{array}\right]
$$

- modelo combinado como em (5),

$$
Y_{j}=X_{j} W_{j} \gamma+X_{j} \nu_{j}+\epsilon_{j}, \quad j=1,2, \ldots, J
$$


em que: $A_{j}=X_{j} W_{j}$, isto é:

$$
A_{j}=\left[\begin{array}{cccc}
1 & W_{j} & X_{1 j} & W_{j} X_{1 j} \\
1 & W_{j} & X_{1 j} & W_{j} X_{1 j} \\
1 & W_{j} & X_{1 j} & W_{j} X_{1 j} \\
\cdots & \cdots & \cdots & \cdots \\
1 & W_{j} & X_{1 j} & W_{j} X_{1 j}
\end{array}\right]
$$

em que $A_{j}$ e $W_{j}$ são as matrizes do delineamento, $\gamma$ é o vetor de efeitos fixos, $\nu_{j}$ é o vetor de efeitos aleatórios e $\epsilon_{j}$ é o vetor de resíduos. As suposições para esse modelo são:

$$
\begin{gathered}
\epsilon_{j} \sim N(0, R), \quad R_{j}=\sigma^{2} I_{n j} \\
\nu_{j} \sim N(0, G), \quad G=\left[\begin{array}{cc}
\tau_{00} & \tau_{01} \\
\tau_{01} & \tau_{11}
\end{array}\right]
\end{gathered}
$$

As variáveis explanatórias $X$ e $W$ para os níveis 1 e 2, respectivamente, podem também ser consideradas em suas medidas originais ou centradas. A partir dessa estrutura, Sullivan et al. (1999) apresentam uma tabela com os casos especiais para os modelos descritos anteriormente.

O modelo (4) não é uma forma na qual o método dos mínimos quadrados ordinários possa ser usado para estimar os parâmetros. Isto porque, nesse tipo de estrutura, vários tipos diferente de parâmetros são usados. Especificamente, os coeficientes $\beta_{j}$ no nível 1 podem ser fixos ou aleatórios; no nível 2 , os coeficientes $\gamma$ são considerados fixos, as variâncias e covariâncias entre os níveis 1 e 2 são chamados componentes de variância. Assim, para estimar os parâmetros envolvidos no modelo, são necessários processos interativos. A seguir, serão abordados tópicos referentes à estimação nos modelos multiníveis. Maiores detalhes teóricos são encontrados em Littell et al. (1996) e em Searle et al. (1992). 


\subsection{Estimação}

Harville (1977) discute a estimação de máxima verossimilhança e a estimação de máxima verossimilhança restrita para modelos de efeitos mistos supondo uma distribuição normal para os dados. Já em Mason et al. (1984), o estimador de máxima verossimilhança restrita é obtido usando o algoritmo EM.

Assim, em (5), se $Y$ é um vetor de observações $n$ x 1 com matriz de variâncias e covariâncias $V$, Goldstein (1986) mostra que, se $V$ é conhecida, então o estimador do parâmetro $\beta$ é dado por:

$$
\hat{\beta}=\left(X^{T} V^{-1} X\right)^{-1} X^{T} V^{-1} Y, \quad \operatorname{cov}(\hat{\beta})=\left(X^{T} V^{-1} X\right)^{-1},
$$

que são os estimadores de mínimos quadrados generalizados usuais.

Se $\beta$ é conhecido mas $V$ é desconhecido, pode-se obter os estimadores $\beta^{*}$ dos parâmetros de $V$ usando novamente o método de mínimos quadrados generalizados como:

$$
\hat{\beta}^{*}=\left(X^{* T}\left(V^{*}\right)^{-1} X^{*}\right)^{-1} X^{* T}\left(V^{*}\right)^{-1} Y^{*}
$$

em que $Y^{*}$ é o vetor dos elementos da matriz triangular superior $(Y-X \beta)(Y-X \beta)^{T}$, que é uma matriz quadrada e produz os resíduos, $V^{*}$ é a matriz de variâncias e covariâncias de $Y^{*}$ e $X^{*}$ é a matriz do delineamento que conduz $Y^{*}$ a $V$ na regressão de $Y^{*}$ em $X^{*}$.

Quando nem $\beta$ e nem $V$ são conhecidos, os estimadores são obtidos de modo que as equações (8) e (9) são satisfeitas simultaneamente, fornecendo, assim, os estimadores de mínimos quadrados generalizados interativos. Ainda, em Goldstein (1986), mostra-se que os estimadores produzidos por esse método são equivalentes aos estimadores de máxima verossimilhança, quando se supõe a distribuição normal para os dados.

O processo de estimação começa com uma estimativa inicial de $V$, usada para obter $\hat{\beta}$, e então obter uma nova estimativa melhorada de $V$ e assim por 
diante, até que a convergência do processo seja obtida.

Sullivan et al. (1999) mostram o processo de estimação do vetor de parâmetros para os efeitos fixos $\gamma$ e para os efeitos aleatórios $\nu$ de um modelo multinível considerando dois níveis. Assim, para o modelo combinado (7), a estimação dos efeitos fixos pode ser feita utilizando o método dos mínimos quadrados ponderados ou por mínimos quadrados generalizados, dado por:

$$
\hat{\gamma}=\left(A^{T} \hat{V}^{-1} A\right)^{-1} A^{T} \hat{V}^{-1} Y
$$

com

$$
V=\operatorname{var}(Y)=X G X^{T}+R
$$

em que $A$ é uma matriz $N \mathrm{x} 4 \operatorname{com} N=\sum_{j-1}^{J}$ e $\hat{V}$ é a matriz $V$ com $G$ e $R$ substituídos pelas seus respectivos estimadores de máxima verossimilhança, obtidos maximizando o logaritmo da função de verossimilhança, dado por:

$$
l_{M V}(G, R)=-\frac{1}{2} \log |V|-\frac{N}{2} \log \left(r^{T} V^{-1} r\right)-\frac{N}{2}\left[1+\log \frac{2 \pi}{N}\right]
$$

em que

$$
r=Y-A\left(A^{T} V^{-1} A\right)^{T} A^{T} V^{-1} Y .
$$

Os elementos de $G$ e $R$ são chamados componentes de variância. Novamente, tem-se que o método de estimação é utilizado interativamente, satisfazendo simultaneamente às equações (10) e (11), para um valor inicial de $V$, até o processo convergir.

Se o número $J$ de unidades do nível 2 é grande, então, os estimadores gerados pela máxima verossimilhança são aproximadamente iguais aos gerados pela máxima verossimilhança restrita. Os estimadores de máxima verossimilhança restrita para os componentes de covariância são baseados nos resíduos, os quais são obtidos após a estimativa dos efeitos fixos (10) através métodos dos mínimos quadrados 
ponderados ou mínimos quadrados generalizados. Nota-se que os estimadores de máxima verossimilhança restrita levam em conta os números de graus de liberdade usados na estimativa dos efeitos fixos ao se estimarem os componentes da covariância.

\subsection{Testes de Hipóteses}

\subsubsection{Teste de Hipótese para os efeitos fixos $\gamma$}

A hipótese de interesse neste caso é da forma $H_{0}: \gamma_{k}=0$. A estatística de teste é calculada considerando o estimador de máxima verossimilhança ou a máxima verossimilhança restrita para o erro padrão, dado por:

$$
t=\frac{\hat{\gamma}_{k}}{\sqrt{v \hat{a} r(\hat{\gamma})}}
$$

A estatística dada em (12) segue uma distribuição $t$-Student para dados balanceados e para algumas situações de desbalanceamento. Na maioria das situações, a distribuição de (12) é aproximada, bem como os graus de liberdade.

\subsubsection{Teste de Hipótese para os componentes de variância $R$ e $G$}

Aqui a hipótese de interesse é da forma $H_{0}: \tau_{k l}=0$, em que $\tau_{k l}$ é um elemento de $G$. A estatística de teste é calculada considerando o estimador de máxima verossimilhança ou a máxima verossimilhança restrita para o erro padrão, dado por:

$$
Z=\frac{\hat{\tau}_{k l}}{\sqrt{v \hat{a} r\left(\hat{\tau}_{k l}\right)}}
$$

O erro padrão é calculado através da segunda derivada da função de verossimilhança com relação aos componentes de variância.

Sullivan et al. (1999) ainda mostram um teste para os efeitos aleatórios $\nu$. 


\subsection{Análise de dados de medidas repetidas}

Dados de medidas repetidas surgem em vários contextos e em todas as áreas do conhecimento. Segundo Singer \& Andrade (1986), nos estudos longitudinais as observações de um conjunto de indivíduos (plantas, animais, pessoas, etc) são classificados em diferentes tratamentos segundo um ou mais fatores, (variedade, raça, sexo, etc) ao longo de diversas ocasiões de medida (tempo, doses, distância, etc). No caso de considerar o tempo como a dimensão ao longo da qual as respostas são observadas, é importante a maneira pela qual os dados são obtidos, ou seja, o número de ocasiões em que cada indivíduo é observado. Assim, podem ocorrer três tipos de estudo:

- cross-sectional - em que se observa cada indivíduo uma única vez,

- longitudinal - em que cada indivíduo é observado em todas as ocasiões,

- misto ou do tipo linked cross-sectional - em que diferentes indivíduos são observados em diferentes subconjuntos de ocasiões. Por exemplo, em um experimento cujo objetivo é avaliar o efeito de um tratamento no crescimento de plantas durante 6 semanas, algumas plantas seriam observadas após 1, 2 e 3 semanas, outras após 3, 4 e 5 semanas e outras após 4, 5 e 6 semanas.

O ensaio em cross-sectional é o de maior simplicidade, não havendo necessidade de acompanhar os indivíduos ao longo das ocasiões de medida, já que o acompanhamento aumenta o custo de um ensaio, quer no aumento do tempo de execução deste ou na necessidade de se evitar perdas de indivíduos, o que ocasionaria uma perda de eficiência na análise. No entanto esse tipo de estudo não leva em consideração as informações sobre a variação individual ao longo do tempo, o que pode levar a um confundimento entre o efeito do tempo e o efeito de grupo.

Já o estudo longitudinal permite incorporar as informações sobre as variações individuais ao longo do tempo, produzindo estimadores mais eficientes 
dos parâmetros associados às medidas de tal variação. O estudo misto também permite incorporar alguma informação sobre o padrão individual de variação, mas a informação sobre outras variáveis (covariáveis) que possam influenciar a resposta, deve ser obtida retrospectivamente, sem possibilidade de controle.

Outro fator que diferencia dados provenientes do estudo tipo crosssectional e dados obtidos com estudo longitudinal é a estrutura de covariância, ou seja, no primeiro há uma total independência entre as observações enquanto que, no longitudinal, espera-se alguma correlação entre as observações realizadas no mesmo indivíduo. Assim grande parte do esforço para analisar esses dados, está relacionada com a modelagem dessa estrutura de covariância, pois o número de parâmetros para descrever essa estrutura de covariância torna-se grande, principalmente se o número de ocasiões de medida aumenta é desbalanceado (os indivíduos não são observados num mesmo número de ocasiões de medida). A perda de indivíduos também pode ocorrer nos estudos longitudinais, seja por razões aleatórias ou por imposição do próprio estudo.

A estrutura básica dos dados em um estudo longitudinal pode ser apresentada como na Tabela 1, em que:

$Y_{i j k}$ é a variável resposta do $j$-ésimo indivíduo submetido ao $i$-ésimo tratamento sob a $k$-ésima ocasião de medida, 
Tabela 1. Estrutura básica dos dados para estudos longitudinais

\begin{tabular}{cccccc}
\hline Tratamentos & Indivíduo & \multicolumn{5}{c}{ Ocasiões de medida } & $(\mathrm{k})$ \\
$(\mathrm{i})$ & $(\mathrm{j})$ & 1 & 2 & $\ldots$ & $\mathrm{t}$ \\
\hline 1 & 1 & $Y_{111}$ & $Y_{112}$ & $\ldots$ & $Y_{11 t}$ \\
1 & 2 & $Y_{121}$ & $Y_{122}$ & $\ldots$ & $Y_{12 t}$ \\
$\vdots$ & $\vdots$ & $\vdots$ & $\vdots$ & & $\vdots$ \\
1 & $n_{1}$ & $Y_{1 n_{1} 1}$ & $Y_{1 n_{1} 2}$ & $\ldots$ & $Y_{1 n_{1} t}$ \\
2 & 1 & $Y_{211}$ & $Y_{212}$ & $\ldots$ & $Y_{21 t}$ \\
2 & 2 & $Y_{221}$ & $Y_{222}$ & $\ldots$ & $Y_{22 t}$ \\
$\vdots$ & $\vdots$ & $\vdots$ & $\vdots$ & & $\vdots$ \\
2 & $n_{2}$ & $Y_{2 n_{2} 1}$ & $Y_{2 n_{2} 2}$ & $\ldots$ & $Y_{2 n_{2} t}$ \\
$\vdots$ & $\vdots$ & $\vdots$ & $\vdots$ & & $\vdots$ \\
$\mathrm{g}$ & 1 & $Y_{g 11}$ & $Y_{g 12}$ & $\ldots$ & $Y_{g 1 t}$ \\
$\mathrm{~g}$ & 2 & $Y_{g 21}$ & $Y_{g 22}$ & $\ldots$ & $Y_{g 2 t}$ \\
$\vdots$ & $\vdots$ & $\vdots$ & $\vdots$ & & $\vdots$ \\
$\mathrm{g}$ & $n_{g}$ & $Y_{g n_{g} 1}$ & $Y_{g n_{g} 2}$ & $\ldots$ & $Y_{g n_{g} t}$ \\
\hline
\end{tabular}

Em termos gerais, o problema de análise de dados longitudinais, consiste na redução do número de parâmetros envolvidos (médias e covariâncias associadas aos $Y_{i j k}$ ) através da imposição de modelos estruturais que relacionam sua variação com os tratamentos e com as ocasiões de medida.

Um modelo simples, considerando apenas os indivíduos e as ocasiões de medida pode ser escrito como segue:

$$
Y_{k j}=\beta_{0}+\sum_{k=1}^{t} \beta_{k} X_{k j}+\epsilon_{k j},
$$

Considerando um modelo multinível, a estrutura do estudo longitudi- 
nal pode ser vista como uma hierarquia de 2 níveis de maneira invertida, na qual as ocasiões de medida são as unidades do nível 1 e os indivíduos como as unidades de nível 2, não havendo necessidade de um mesmo número de ocasiões de medidas por indivíduo (Rasbash et al., 2001). Assim:

$$
Y_{i j}=\beta_{0 j}+\sum_{i=1}^{n_{j}} \beta_{i} X_{i j}+\epsilon_{i j}
$$

\subsection{Softwares}

Tradicionalmente, softwares para análise estatística de modelo linear ou modelos lineares generalizados assumem um modelo de um único nível com uma única variável aleatória. Para os modelos multiníveis tais softwares são inadequados, o que gerou, a partir de 1980, o desenvolvimento de quatro softwares para ajustar modelos multiníveis: GENMOD (Mason et al., 1988), HLM (Bryk et al., 1988), VARCL (Longford, 1988) e MLwiN (Rasbash et al., 2001). Uma revisão detalhada destes quatro pacotes (inclusive o ML3 que subseqüentemente se tornou MLn e então MLwiN) foi feita por Kreft et al. (1994), bem como por Zhou et al. (1999), numa comparação dos programas HLM, VARCL, MLwiN e o PROC MIXED do sistema SAS (Statistical Analysis System).

Na sua forma original o HLM, ML3 e VARCL foram planejados para variáveis respostas contínuas, normalmente distribuídas considerando métodos de estimação de máxima verossimilhança ou máxima verossimilhança restrita. Esses três programas ajustam modelos com uma estrutura complexa de variância, para até três níveis. O VARCL bem como o ML3 têm procedimentos para ajustar modelos de resposta Binomial ou Poisson. Subseqüentemente, os programas estatísticos de maior uso, principalmente BMDP, SAS e GENSTAT, incorporaram procedimentos para estimativas de máxima verossimilhança e de máxima verossimilhança restrita. Os programas EGRET e SABRE permitem a obtenção de estimativas de máxima verossimilhança para um modelo logit de 2 níveis. Os programas MLn e MLwiN 
permitem um número efetivamente ilimitado de níveis, juntamente com estimativas robustas de erros padronizados.

\subsubsection{Programa MLwiN}

O Programa MLwiN, versão 1.10, faz parte do Multilevel Models Project do Instituto de Educação da Universidade de Londres.

Este programa é desenvolvido para Windows 95/98 ou Windows NT/2000. A estrutura dos dados é a mesma de uma planilha ou de um arquivo, com as colunas denotando as variáveis e as linhas representando as unidades de níveis mais baixo da hierarquia. Como padrão o programa aloca 400 colunas, 150 variáveis explanatórias e 5 níveis de hierarquia, sendo que as dimensões do arquivo de trabalho, o número de parâmetros e o número de níveis podem ser alterados dinamicamente. Este programa, até esta versão, não importa dados de outros programas, a leitura do conjunto de dados é feita pela leitura de um conjunto ASCII ou utilizando-se o comando copiar/colar. As variáveis alfanuméricas devem ser substituídas por números e também é necessário utilizar um código numérico para dados perdidos, como por exemplo -9999.

Os dados devem ser ordenados de acordo com a hierarquia, ou seja, para uma estrutura de 2 níveis, é necessário incluir as unidades do nível 1 para a primeira unidade do nível 2, seguidas das unidades do nível 1 para a segunda unidade do nível 2 e assim sucessivamente. No caso de modelos com classificação cruzada haverá a necessidade de arranjos mais complexos.

Os dois principais procedimentos de estimação utilizados pelo MLwiN são o método dos Mínimos Quadrados Generalizados Interativos (Interative Generalized Least Squares - IGLS) e o método Monte Carlo para Cadeias de Markov (Monte Carlo Markov Chain - MCMC), sendo que, para dados com resposta discreta, são calculadas estimativas de quasi-verossimilhança. Quanto à inferência, o programa utiliza testes de significância e intervalos de confiança para grandes amostras e o 
método bootstrap para a correção de viés na estimativa de pequenas amostras e erros de medida (Rasbash et al., 2001).

\subsubsection{PROC MIXED do SAS}

Singer (1998) comenta que o PROC MIXED do SAS é um programa flexível para o ajuste de modelos multiníveis. Isso pelo fato desse programa ter sido desenvolvido para analisar modelos mistos, em que são levandos em conta efeitos fixos e aleatórios, permitindo considerar interceptos e inclinações como efeitos aleatórios e medir a variabilidade existente nos níveis e entre níveis. Um problema também citado pela autora, é o fato de que o usuário pode não estar familiarizado com a linguagem SAS. Assim, uma descrição detalhada da aplicação do PROC MIXED para modelos multiníveis pode ser encontrada no artigo. 


\section{METODOLOGIA}

\subsection{Análise dos dados do Programa de Atenção ao Idoso - "PAI"}

\subsubsection{Descrição do experimento}

O primeiro conjunto de dados foi coletado de um Programa de Atenção ao Idoso (PAI), criado pelo Ministério da Saúde e com implementação facultativa pelos municípios. A Secretaria Municipal de Saúde de Alfenas, M.G., implementou o PAI no Ambulatório Dr. Plínio do Prado Coutinho em 1999, tendo já passado por algumas reformulações e atualmente consta de equipe formada por: médico geriatra, enfermeira, assistente social, fisioterapeuta e técnico de enfermagem. Junto a esta equipe prestam assistência na qualidade de participantes consultores fonoaudiólogo, odontólogo, psicólogo, terapeuta ocupacional e nutricionista.

As atividades do programa são desenvolvidas no período da manhã, de segunda à sexta-feira, atendendo todos os clientes referenciados das Unidades Básicas de Saúde, com idade igual ou superior à 60 anos .

Como protocolo é realizada avaliação multidimensional destes clientes, ou seja os aspectos físicos, estrutura de suporte social, estado psicológico e habilidade funcional. Todos os clientes passam obrigatoriamente pela consulta médica e de enfermagem, e pelo assistente social, sendo encaminhados aos demais profissionais quando necessário. Além deste atendimento são realizadas visitas domiciliares a todo cliente que inicia o Programa, podendo repetir-se sempre que houver necessidade. 
Os dados obtidos pelo "PAI" referem-se ao sexo, raça e estado civil dos idosos e as medidas de pressão arterial, peso e altura tomados a cada consulta, no período entre Março de 2000 à Dezembro de 2001, num total de 22 meses (ocasiões). Como o retorno à cada consulta não ocorre todos os meses, isto leva a uma ausência de informação mensal de cada idoso, bem como, dos 166 idosos pesquisados apenas 18 encontravam-se no programa em Março de 2000 e outros possivelmente podem ter abandonado o programa, gerando um número grande de observações faltantes.

Para a utilização de um modelo multinível, considerou-se as ocasiões da consulta como variável de nível 1 e os idosos de nível 2. Como variáveis resposta, considerou-se a pressão arterial sistólica e diastólica tomadas sentado e em pé e, como variáveis explanatórias, o índice de massa corpórea (IMC, $\mathrm{kg} / \mathrm{m}^{2}$ ), sexo, raça e estado civil. A variável IMC foi considerada centrada no seu valor médio, sendo utilizada a notação IMCC. Assim, o modelo foi estruturado como segue:

Variáveis do nível 1 (ocasião da consulta)

- $\operatorname{pass}_{i j}$ : valor da pressão arterial sistólica sentado na $i$-ésima ocasião para o idoso $j$;

- $\operatorname{pads}_{i j}$ : valor da pressão arterial diastólica sentado na $i$-ésima ocasião para o idoso $j$;

- $\operatorname{pasp}_{i j}$ : valor da pressão arterial sistólica em pé na $i$-ésima ocasião para o idoso $j$;

- $\operatorname{padp}_{i j}$ : valor da pressão arterial diastólica em pé na $i$-ésima ocasião para o idoso $j$;

- imcc $_{i j}$ : valor do índice de massa corpórea centrado na sua média geral para $i$-ésima ocasião no $j$-ésimo idoso. 
Variáveis do nível 2 (idoso)

- $\operatorname{sexo}_{j}= \begin{cases}1 & \text { se o idoso é do sexo feminino } \\ 0 & \text { se o idoso é do sexo masculino }\end{cases}$

- raça $_{j}= \begin{cases}1 & \text { se o idoso é da raça branca } \\ 0 & \text { em caso contrário }\end{cases}$

- estado civil $_{j}= \begin{cases}1 & \text { se o idoso é casado } \\ 0 & \text { em caso contrário }\end{cases}$

Serão conduzidas análises para todas as variáveis resposta obtidas do programa. Assim, como uma notação de forma maneira geral, usaremos pa**, para denotar a pressão arterial. Para cada caso em separado, será utilizada a notação: PASS - pressão arterial sistólica sentado, PADS - pressão arterial diastólica sentado, PASP - pressão arterial sistólica em pé, PADP - pressão arterial diastólica em pé. Para os testes de hipóteses e obtenção dos p-valores, serão utilizadas o teste t-Student para os efeitos fixos, dado por (12), e a aproximação normal para os componentes de variância, dado por (13).

\subsubsection{Descrição dos modelos}

Inicialmente, foi considerado um modelo apenas com os interceptos dado por:

$$
\begin{aligned}
& p a_{i j}^{* *}=\beta_{0 j}+\epsilon_{i j} \\
& \beta_{0 j}=\gamma_{00}+\nu_{0 j},
\end{aligned}
$$

em que

$\epsilon_{i j} \sim N\left(0, \sigma^{2}\right), \epsilon_{i j}$ 's independentes;

$\nu_{0 j} \sim N\left(0, \tau_{00}\right)$ e $\nu_{0 j}$ 's independentes; 
$\operatorname{Cov}\left(\nu_{0 j}, \nu_{1 j}\right)=\tau_{01} \mathrm{e}$

$\nu_{0 j}$ 's independentes dos $\epsilon_{i}$ 's.

Assim, no nível 1, a variável resposta da ocasião, pa** é a soma de um intercepto para os idosos de uma ocasião $\left(\beta_{0 j}\right)$ com um erro aleatório $\left(\epsilon_{i j}\right)$ associado à $i$-ésima ocasião no $j$-ésimo idoso. No nível 2 , o intercepto do nível dos idosos é a soma da média global $\left(\gamma_{00}\right)$ com uma série de desvios aleatórios em relação a essa média $\left(\nu_{0 j}\right)$.

Adicionando-se a seguir, a variável IMCC ao modelo como uma variável explanatória, tem-se:

$$
\begin{aligned}
& p a_{i j}^{* *}=\beta_{0 j}+\beta_{1 j}\left(i m c_{i j}-M\right)_{i j}+\epsilon_{i j} \\
& \beta_{0 j}=\gamma_{00}+\nu_{0 j} \\
& \beta_{1 j}=\gamma_{10},
\end{aligned}
$$

em que

$M$ é a média geral da variável $i m c_{i j}$;

$\epsilon_{i j} \sim N\left(0, \sigma^{2}\right), \epsilon_{i j}$ 's independentes;

$\nu_{0 j} \sim N\left(0, \tau_{00}\right)$ e $\nu_{0 j}$ 's independentes;

$\nu_{0 j}$ 's independentes dos $\epsilon_{i}$ 's.

Com a variável explanatória do nível 1 centrada na média geral imcc $=$ $\left(\right.$ imc $\left._{i j}-M\right)$, tem-se a mudança esperada na $\mathrm{pa}^{* *}$ do $j$-ésimo idoso quando o IMC aumenta de uma unidade em relação à sua média geral $M$. Assim, o intercepto $\beta_{0 j}$ é a pa** esperada no $j$-ésimo idoso quando ela atinge um IMC igual a $M$.

Considerando, a seguir, o IMCC com efeito aleatório para o nível dos idosos, ou seja, não apenas a pressão em uma determinada ocasião esta relacionada com um IMCC dessa ocasião, mas também a relação entre os IMCC pode variar através dos idosos, tem-se: 


$$
\begin{aligned}
& p a_{i j}^{* *}=\beta_{0 j}+\beta_{1 j}\left(i m c_{i j}-M\right)_{i j}+\epsilon_{i j} \\
& \beta_{0 j}=\gamma_{00}+\nu_{0 j} \\
& \beta_{1 j}=\gamma_{10}+\nu_{1 j},
\end{aligned}
$$

em que

$M$ é a média geral da variável $i m c_{i j}$;

$\epsilon_{i j} \sim N\left(0, \sigma^{2}\right), \epsilon_{i j}$ 's independentes;

$\nu_{0 j} \sim N\left(0, \tau_{00}\right)$ e $\nu_{0 j}$ 's independentes;

$\nu_{1 j} \sim N\left(0, \tau_{11}\right)$ e $\nu_{1 j}$ 's independentes;

$\operatorname{Cov}\left(\nu_{0 j}, \nu_{1 j}\right)=\tau_{01} \mathrm{e}$

$\nu_{0 j}$ 's e $\nu_{1 j}$ 's independentes dos $\epsilon_{i}$ 's.

O modelo (18), permite ao intercepto e a inclinação variarem nos idosos, aumentando a matriz de variâncias e covariâncias para representar os efeitos aleatórios nos idosos, isto é, os componentes de variância do intercepto e da inclinação são adicionados de um componente de covariância entre o intercepto e a inclinação $\left(\tau_{01}\right)$. A matriz de covariância pode ser representada de uma forma simples, como:

$$
\left(\begin{array}{ll}
\hat{\tau}_{00} & \hat{\tau}_{01} \\
\hat{\tau}_{10} & \hat{\tau}_{11}
\end{array}\right) .
$$

Adicionando as variáveis explanatórias do segundo nível: sexo, raça e estado civil ao modelo (18), tem-se:

$$
\begin{aligned}
& p a_{i j}^{* *}=\beta_{0 j}+\beta_{1 j} \text { imcc }+\beta_{2} \text { masculino }_{j}+\beta_{3} \text { não } \text { branco }_{j}+\beta_{4} \text { não casado } j+\epsilon_{i j} \\
& \beta_{0 j}=\gamma_{00}+\nu_{0 j} \\
& \beta_{1 j}=\gamma_{10}+\nu_{1 j}
\end{aligned}
$$

em que as suposições dos efeitos aleatórios são análogas às do modelo (18). 


\subsection{Análise dos dados do teor de proteína no leite}

\subsubsection{Descrição do experimento}

O segundo conjunto de dados, retirado do livro de Diggle et al. (1994), refere-se a um experimento em que foi medido o teor de proteína no leite de 79 vacas australianas, coletados durante 19 semanas e submetidas a três dietas: cevada (25 vacas), uma mistura de cevada e tremoço (27 vacas) e apenas o tremoço (27 vacas). Essas medidas repetidas em cada animal acentuam a natureza longitudinal desse conjunto de dados.

As medidas do teor de proteína no leite foram feitas a partir do parto, sendo que o experimento termina após 19 semanas do primeiro parto, o que torna incompleta metade das 79 medidas efetuadas. Portanto, se o parto está associado direta ou indiretamente ao processo fisiológico que determina o teor de proteínas no leite, essas observações faltantes não podem ser ignoradas.

Neste exemplo, para a utilização de um modelo multinível, foi considerado as observações no nível 1 e os animais no nível 2; como variável resposta tem-se o teor de proteína no leite e como variáveis explanatórias, o tempo (em semanas) e a dieta (cevada, tremoço, e uma mistura de cevada e tremoço).

\subsubsection{Descrição dos modelos}

A análise para a variável teor de proteína no leite, inicialmente, seguirá os modelos análogos aos (16), (17) e (18), em que a variável explanatória TEMPO não está centrada na média geral $\left(\beta_{1 j}\right.$ tempo $\left.o_{i j}\right)$. Assim, tem-se a mudança esperada no TEOR quando o TEMPO aumenta de uma unidade (semana) e o intercepto $\beta_{0 j}$ é o TEOR esperado quando o TEMPO é igual a zero.

Incluindo a variável explanatória do segundo nível, a dieta, um modelo semelhante ao (16) é dado através do modelo (17). 


$$
\begin{aligned}
& \text { teor }_{i j}=\beta_{0 j}+\beta_{1 j} \text { tempo }+ \text { beta }_{2} \text { mistura }_{j}+\beta_{3} \text { tremoço }_{j}+\epsilon_{i j} \\
& \beta_{0 j}=\gamma_{00}+\nu_{0 j} \\
& \beta_{1 j}=\gamma_{10}+\nu_{1 j} .
\end{aligned}
$$

Incluindo-se, agora as interações das dietas com o tempo ao modelo (20), tem-se o modelo (21).

$$
\begin{aligned}
\text { teor }_{i j}= & \beta_{0 j}+\beta_{1 j} \text { tempo }+\beta_{2} \text { mistura }_{j}+\beta_{3} \text { tremoço }_{j}+\beta_{4} \text { mistura }^{*} \text { tempo }_{j} \\
+ & \beta_{5} \text { tremoço }^{*} \text { tempo }_{j}+\epsilon_{i j} \\
& \beta_{0 j}=\gamma_{00}+\nu_{0 j} \\
& \beta_{1 j}=\gamma_{10}+\nu_{1 j} .
\end{aligned}
$$

Os exemplos serão analisados através dos softwares MLwiN, versão 1.10 e do SAS versão 8.00. Para o primeiro conjunto de dados, usando o software MLwiN, após a transferência do arquivo (Excel) já com um valor para a falta de observações $(-999,00)$, tem-se que a disposição dos dados não reflete uma estrutura hierárquica com repetidas medidas em cada um dos idosos, assim torna-se necessário a utilização do item Split Records da opção Data Manipulation na barra de menus. Já para o SAS, utilizou-se do arquivo gerado no MLwiN, através do item ASCII TEXT FILE OUTPUT da opção File na barra de menus, salvo em um arquivo .TXT.

No segundo conjunto de dados, o experimento é analisado em Diggle et al. (1994) e novamente reanalisado no software Oswald (Object-oriented Software for the Analysis of Longitudinal Data in S-PLUS), versão 3.2, do qual os dados foram obtidos (Smith, 1997). 


\section{RESULTADOS E DISCUSSÃO}

\subsection{Resultados da análise dos dados do "PAI"}

Considerando a variável pressão arterial sistólica sentado, $\operatorname{pass}_{i j}$, e seguindo o modelo dado em (16), as estimativas dos parâmetros considerados são mostradas na Tabela 2.

Tabela 2. Estimativa dos parâmetros do modelo (16) considerando apenas os interceptos para os dados de pressão arterial sistólica sentado - "PAI" .

\begin{tabular}{ccrc}
\hline Parâmetros & Estimativas & Erro Padrão & p-valor \\
\hline$\gamma_{00}$ & 142,577 & 1,333 & $<0,0001$ \\
$\tau_{00}$ & 237,840 & 32,203 & $<0,0001$ \\
$\sigma^{2}$ & 315,303 & 14,178 & $<0,0001$ \\
Estatística & $-2^{*}$ log-veros. & 10201,950 & - \\
\hline
\end{tabular}

Neste modelo foi considerado o intercepto como um efeito fixo. A estimativa de 142,577 mmHg dada na Tabela 2, representa a pressão média arterial sistólica sentado dos idosos. Já, analisando os resíduos considerados como efeitos aleatórios, observa-se uma maior variação entre ocasiões dentro dos idosos $\left(\sigma^{2}=\right.$ $315,303)$ do que apenas entre os idosos $\left(\tau_{00}=237,840\right)$. Isto indica a existência de uma influência das ocasiões na pressão arterial sistólica sentado para cada idoso. 
Uma outra forma de verificar a variação na pressão arterial sistólica sentado seria estimar a correlação intra-grupo (neste caso, intra-idosos), indicada por $\rho$, a qual estima a parte da variação total que ocorre entre as unidades do nível 2 (idosos). Este valor é:

$$
\hat{\rho}=\frac{237,840}{237,840+315,303}=0,4300
$$

e mede até que ponto a pressão arterial sistólica sentado medida nas ocasiões num mesmo idoso assemelha-se quando comparada com as pressões nos diferentes idosos. Tem-se que, neste caso, este valor indica apenas $43 \%$ de variabilidade intra-idosos. Ocorre que o modelo ajustado é bastante simples, podendo incluir outras variáveis medidas no programa obtendo-se um possível aumento nesse valor.

Ainda no modelo considerado, o valor da estatística -2 vezes o logaritmo da função de verossimilhança foi de 10201,950, obtida considerando 1154 valores do total de 3652 observações. Essa estatística será utilizada na comparação desse modelo simples com modelos mais complexos, através de um teste $\chi^{2}$ para a razão das verossimilhança.

Continuando com a variável pressão arterial sistólica sentado, pass $_{i j}$ e seguindo agora o modelo dado em (17), as estimativas dos parâmetros considerados são mostrados na Tabela 3.

Tem-se na Tabela 3, um intercepto de 141,899 $\mathrm{mmHg}$ representando a pressão arterial sistólica sentado esperada no $j$-ésimo idoso quando o IMCC for igual a 25,2482 (valor médio $M$ para a variável). A estimativa da variável IMCC foi positiva $(1,094)$, indicando um aumento da pressão arterial sistólica sentado com o aumento do IMCC, ou seja a pressão arterial sistólica sentado aumenta de 1,094 mmHg, quando o IMCC aumenta de uma unidade em relação à média geral 25,2482. Para os resíduos, observa-se pouca alteração em relação ao modelo considerando apenas os interceptos, ou seja, entre ocasiões dentro dos idosos $\left(\sigma^{2}=309,694\right)$ e entre os idosos $\left(\tau_{00}=234,810\right)$. Isso indica uma influência das ocasiões na pressão arterial sistólica sentado para cada idoso, mesmo após a inclusão da variável IMCC. 
Tabela 3. Estimativa dos parâmetros do modelo (17) considerando IMCC como uma variável explanatória para os dados de pressão arterial sistólica sentado "PAI".

\begin{tabular}{crrc}
\hline Parâmetros & Estimativas & Erro Padrão & p-valor \\
\hline$\gamma_{00}$ & 141,899 & 1,348 & $<0,0001$ \\
$\gamma_{01}$ & 1,094 & 0,260 & $<0,0001$ \\
$\tau_{00}$ & 234,810 & 32,137 & $<0,0001$ \\
$\sigma^{2}$ & 309,694 & 14,012 & $<0,0001$ \\
Estatística & $-2^{*}$ log-veros. & 10039,010 & - \\
\hline
\end{tabular}

Neste caso a correlação intra-idosos é dada por:

$$
\hat{\rho}=\frac{234,810}{234,810+309,694}=0,4312
$$

o que praticamente não muda em relação ao modelo anterior.

A estatística -2 vezes o logaritmo da função de verossimilhança para esse modelo é igual a 10039,010, considerando 1138 valores do total de 3652 observações. Isto mostra uma redução do modelo com a variável IMCC em relação ao modelo só com os interceptos $(10201,950$ - 10039,010 = 162,94). Assim, o valor da diferença obtida entre os dois modelos se com parado com o quantil de uma distribuição $\chi^{2}$ com 1 grau de liberdade, tem-se que uma redução significativa nessa estatística $(p<0,0001)$. Assim, verifica-se que há uma variação da pressão arterial de idoso para idoso com diferentes IMCC.

Fazendo o ajuste para a variável $\operatorname{pass}_{i j}$ no modelo (18), ou seja, fazendo a variável IMCC agora como um efeito aleatório para o nível dos idosos, os resultados são mostrados na Tabela 4.

De acordo com a Tabela 4, a pressão arterial sistólica sentado dos idosos controlada pelo IMCC das ocasiões é de 142,019 e o valor 1,118 é a inclinação média representando a relação entre essa pressão e o IMCC das ocasiões. 
Tabela 4. Estimativa dos parâmetros do modelo (18) considerando o IMCC com efeito aleatório para o nível dos idosos para os dados de pressão arterial sistólica sentado - "PAI".

\begin{tabular}{crrr}
\hline Parâmetros & Estimativas & Erro Padrão & p-valor \\
\hline$\gamma_{00}$ & 142,019 & 1,329 & $<0,0001$ \\
$\gamma_{10}$ & 1,118 & 0,266 & $<0,0001$ \\
$\tau_{00}$ & 227,804 & 37,072 & $<0,0001$ \\
$\tau_{11}$ & 0,147 & 0,918 & 0,8728 \\
$\tau_{01}$ & 3,103 & 3,374 & 0,3577 \\
$\sigma^{2}$ & 309,506 & 14,016 & $<0,0001$ \\
Estatística & $-2^{*} \log$-veros. & 10038,000 & - \\
\hline
\end{tabular}

A matriz de covariância é dada por:

$$
\left(\begin{array}{ll}
\hat{\tau}_{00} & \hat{\tau}_{01} \\
\hat{\tau}_{10} & \hat{\tau}_{11}
\end{array}\right)=\left(\begin{array}{cc}
227,804 & 0,147 \\
0,147 & 3,103
\end{array}\right) .
$$

O valor 227,804 representa a variação nos interceptos, enquanto que o valor 3,103 representa a variação nas inclinações e 0,147 representa a covariância entre os interceptos e as inclinações. Neste caso, nota-se que existe uma grande variabilidade nos interceptos. Em outras palavras, as pressões arteriais sistólicas sentado dos idosos diferem, em média, mesmo após a inclusão do efeito IMCC. Já, as inclinações não tem o mesmo significado, uma vez que apresentaram um erro padrão alto $(0,918)$. Nota-se também que a covariância entre os interceptos e as inclinações é muito pequena em relação ao seu erro padrão $(3,374)$. Assim, não há evidência de que os efeitos dos IMCC's das ocasiões sobre as diferentes valores de pressão arterial sistólica sentado difiram, dependendo da pressão arterial sistólica sentado média no idoso.

Para o ajuste do modelo (18), o valor da estatística -2 vezes o logaritmo da função de verossimilhança foi de 10038,000 considerando 1138 valores de um total 
de 3652 observações. Isto indica uma pequena redução do modelo, considerando a variável IMCC como efeito aleatório para o nível dos idosos em relação ao modelo considerando a variável IMCC apenas como explanatória fixa (10039,010 - 10038,000 $=1,010)$. Comparando o valor da diferença das estatísticas com o quantil de uma distribuição $\chi^{2}$ com 2 graus de liberdade, tem-se que o p-valor é de 0,6035, comprovando a não significância dos modelos ajustados. Desse modo, quando se considera as inclinações como efeitos aleatórios, não existe uma melhora no modelo, indicando que não existe uma variação da pressão arterial sistólica sentado de idoso para idoso através das ocasiões.

Adicionando, agora, as variáveis explanatórias do segundo nível, ou seja, o sexo, a raça e o estado civil, os resultados para a variável pass ${ }_{i j}$, com o modelo (19), são mostrados na Tabela 5.

Tabela 5. Estimativa dos parâmetros do modelo (19) considerando o IMCC com efeito aleatório para o nível dos idosos mais as variáveis explanatórias desse nível para os dados de pressão arterial sistólica sentado - "PAI" .

\begin{tabular}{crrr}
\hline Parâmetros & Estimativas & Erro Padrão & p-valor \\
\hline$\gamma_{00}$ & 142,568 & 2,164 & $<0,0001$ \\
$\gamma_{10}$ & 1,146 & 0,275 & $<0,0001$ \\
$\beta_{2}$ & 0,633 & 2,988 & 0,8325 \\
$\beta_{3}$ & $-4,301$ & 3,088 & 0,1656 \\
$\beta_{4}$ & 0,933 & 2,753 & 0,7351 \\
$\tau_{00}$ & 227,635 & 37,270 & $<0,0001$ \\
$\tau_{11}$ & 0,272 & 0,954 & 0,7756 \\
$\tau_{01}$ & 3,306 & 3,459 & 0,3392 \\
$\sigma^{2}$ & 309,210 & 14,011 & $<0,0001$ \\
Estatística & $-2^{*} \log$-veros. & 10036,030 & - \\
\hline
\end{tabular}

Analisando a Tabela 5, nota-se que pouca alteração ocorreu nas esti- 
mativas dos parâmetros, não alterando as conclusões obtidas para a variável resposta $\operatorname{pass}_{i j}$ considerando o modelo (18), mesmo após a inclusão das variáveis explanatórias do nível dos idosos. Isto pode ser observado pelo valor da estatística -2 vezes o logaritmo da função de verossimilhança, que apresenta uma pequena redução nos valores $(10038,000-10036,030=1,970)$. Novamente, se comparar esse valor obtido com o quantil de uma distribuição $\chi^{2}$ com 3 graus de liberdade, tem-se um p-valor de 0,5787, indicando a não significância para o modelo adicionado das variáveis sexo, raça e estado civil para o nível dos idosos.

Portanto, o modelo final a ser considerado para a variável $\operatorname{pass}_{i j}$ leva em consideração apenas a variável explanatória IMCC fixa, de acordo com o modelo (17).

A Figura 5 representa graficamente o valor estimado da variável pass ${ }_{i j}$, de acordo com o modelo (17), na qual observa-se diferenças nos interceptos e igualdade nas inclinações.

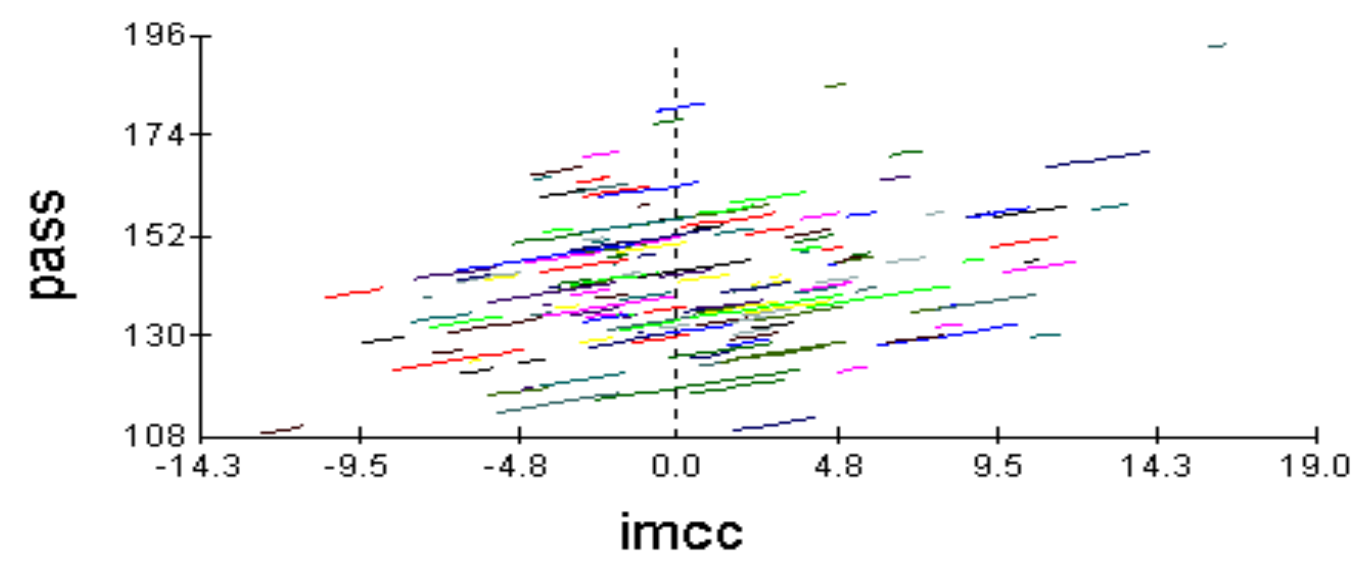

Figura 5 - Gráfico do modelo final para a variável $\operatorname{pass}_{i j}$ 
Considerando agora, a variável pressão arterial diastólica sentado, $\operatorname{pads}_{i j}$, e seguindo o modelo dado em (16), as estimativas dos parâmetros considerados são mostrados na Tabela 6 .

Tabela 6. Estimativa dos parâmetros do modelo (16) considerando apenas os interceptos para os dados de pressão arterial diastólica sentado - "PAI" .

\begin{tabular}{crrc}
\hline Parâmetros & Estimativas & Erro Padrão & p-valor \\
\hline$\gamma_{00}$ & 84,259 & 0,645 & $<0,0001$ \\
$\tau_{00}$ & 50,951 & 7,504 & $<0,0001$ \\
$\sigma^{2}$ & 101,632 & 4,568 & $<0,0001$ \\
Estatística & $-2 * \log$-veros. & 8842,979 & - \\
\hline
\end{tabular}

A estimativa de 84,259 mmHg dada na Tabela 6, representa a média da pressão arterial diastólica sentado dos idosos. Nos resíduos, a maior variação continua entre ocasiões dentro dos idosos $\left(\sigma^{2}=101,632\right)$ do que apenas entre os idosos $\left(\tau_{00}=\right.$ 50,951), indicando uma influência das ocasiões na pressão arterial diastólica sentado para cada idoso.

A estimativa de $\rho$ dada por

$$
\hat{\rho}=\frac{50,951}{50,951+101,632}=0,3339
$$

indica apenas 33,39\% de variabilidade intra-idosos.

Incluindo-se agora ao modelo (16) a variável IMCC, tem-se as estimativas dos parâmetros dadas na Tabela 7, através do modelo (17). 
Tabela 7. Estimativa dos parâmetros do modelo (17) considerando IMCC como uma variável explanatória para os dados de pressão arterial diastólica sentado - "PAI" .

\begin{tabular}{crrc}
\hline Parâmetros & Estimativas & Erro Padrão & p-valor \\
\hline$\gamma_{00}$ & 83,815 & 0,635 & $<0,0001$ \\
$\gamma_{01}$ & 0,579 & 0,125 & $<0,0001$ \\
$\tau_{00}$ & 46,797 & 7,095 & $<0,0001$ \\
$\sigma^{2}$ & 100,356 & 4,538 & $<0,0001$ \\
Estatística & $-2^{*}$ log-veros. & 8695,799 & - \\
\hline
\end{tabular}

O intercepto igual a $83,815 \mathrm{mmHg}$ representa a pressão arterial diastólica sentado esperada no $j$-ésimo idoso quando o IMCC for igual a 25,2482 (valor médio $M$ para a variável). A estimativa positiva para a variável IMCC $(0,579)$, indica um aumento da pressão arterial diastólica sentado com o aumento do IMCC, ou seja a pressão arterial diastólica sentado aumenta de 0,579 $\mathrm{mmHg}$, quando o IMCC aumenta de uma unidade em relação à média geral 25,2482. Para os resíduos, novamente ocorre pouca alteração em relação ao modelo apenas com os interceptos, entre ocasiões dentro dos idosos $\left(\sigma^{2}=100,356\right)$ e entre os idosos $\left(\tau_{00}=46,797\right)$, indicando uma influência das ocasiões na pressão arterial diastólica sentado para cada idoso, mesmo após a inclusão da variável IMCC. Já a correlação intra-idosos dada por:

$$
\hat{\rho}=\frac{46,797}{46,797+100,356}=0,3180
$$

que possui uma pequena redução em relação ao modelo anterior.

Para esse caso, a estatística -2 vezes o logaritmo da função de verossimilhança $(8695,799)$, considerando os 1138 valores do total de 3652 observações, mostra uma redução em relação ao modelo anterior $(8842,979$ - 8695,799 =147,180), valor este que, comparado ao quantil de uma distribuição $\chi^{2}$ com 1 grau de liberdade, fornece um p-valor $<0,0001$, verificando-se uma variação significativa da pressão 
arterial de idoso para idoso para os diferentes valores de IMCC.

Considerando-se agora a variável IMCC como aleatória para o nível dos idosos de acordo com o modelo (18), tem-se as estimativas dos parâmetros mostradas na Tabela 8.

Tabela 8. Estimativa dos parâmetros do modelo (18) considerando o IMCC com efeito aleatório para o nível dos idosos para os dados de pressão arterial diastólica sentado - "PAI" .

\begin{tabular}{crrr}
\hline Parâmetros & Estimativas & Erro Padrão & p-valor \\
\hline$\gamma_{00}$ & 83,821 & 0,630 & $<0,0001$ \\
$\gamma_{10}$ & 0,550 & 0,131 & $<0,0001$ \\
$\tau_{00}$ & 43,748 & 8,137 & $<0,0001$ \\
$\tau_{11}$ & 0,119 & 0,215 & 0,5799 \\
$\tau_{01}$ & 1,061 & 0,698 & 0,1285 \\
$\sigma^{2}$ & 100,405 & 4,538 & $<0,0001$ \\
Estatística & $-2^{*} \log$-veros. & 8695,228 & - \\
\hline
\end{tabular}

Pela Tabela 8, a pressão arterial diastólica sentado dos idosos controlada pelo IMCC das ocasiões é de 83,821 mmHg e o valor 0,550 é a inclinação média representando a relação entre essa pressão e o IMCC das ocasiões.

A matriz de covariância estimada é:

$$
\left(\begin{array}{ll}
\hat{\tau}_{00} & \hat{\tau}_{01} \\
\hat{\tau}_{10} & \hat{\tau}_{11}
\end{array}\right)=\left(\begin{array}{cc}
43,748 & 1,061 \\
1,061 & 0,119
\end{array}\right) .
$$

A variação nos interceptos é dada por 43,748, enquanto que 0,119 representa a variação nas inclinações. Já, a covariância entre os interceptos e as inclinações é dada por 1,061. Observa-se, assim, uma grande variabilidade nos interceptos, ou seja, as pressões arteriais diastólica sentado dos idosos, mesmo após a inclusão do efeito do IMCC, diferem, em média. Já, as inclinações não tem o mesmo significado, 
uma vez que apresentaram um erro padrão alto $(0,215)$ em relação ao valor da estimativa do parâmetro considerado $(0,119)$, com um p-valor de 0,5799 , o que indica que esta variação nas inclinações pode ser considerada nula. Além disso, nota-se que a covariância entre os interceptos e as inclinações $(1,061)$ com um erro padrão de 0,698, forneceu um p-valor de 0,1285, indicando, novamente que não existe uma relação entre eles. Desse modo, tem-se, então, uma ausência dos efeitos dos IMCC's das ocasiões sobre as diferentes valores de pressão arterial diastólica sentado no idoso.

O valor da estatística -2 vezes o logaritmo da função de verossimilhança foi 8695,228 de 1138 valores no total de 3652 observações, mostrando uma redução não significativa do modelo com a variável IMCC com efeito aleatório para o nível dos idosos e o modelo considerando a variável IMCC como uma variável explanatória fixa $(8695,799-8695,228=0,571)$, se comparado a uma distribuição $\chi^{2}$ com 2 graus de liberdade $(\mathrm{p}$-valor $=0,6035)$. Assim, tem-se que não existe uma melhora no modelo quando se considera as inclinações como efeitos aleatórios indicando, portanto, que não existe uma variação da pressão arterial diastólica sentado de idoso para idoso através das ocasiões.

Incluindo agora, as variáveis explanatórias do segundo nível, ou seja, o sexo, a raça e o estado civil, tem-se as estimativas dos parâmetros para o modelo (19) apresentadas na Tabela 9.

Analisando a Tabela 9, verifica-se pouca alteração nas estimativas dos parâmetros. Portanto, não há alteração nas conclusões obtidas para a variável resposta $\operatorname{pads}_{i j}$ mesmo após a inclusão das variáveis explanatórias do nível dos idosos, através do modelo (19). Isto pode ser comprovado através da diferença entre as estatísticas -2 vezes o logaritmo da função de verossimilhança (8695,228 - 8691,148 $=4,080)$ com o quantil de uma distribuição $\chi^{2}$ com 3 graus de liberdade ( $\mathrm{p}$-valor $=$ $0,2560)$. 
Tabela 9. Estimativa dos parâmetros do modelo (19) considerando o IMCC com efeito aleatório para o nível dos idosos mais as variáveis explanatórias desse nível para os dados de pressão arterial diastólica sentado - "PAI" .

\begin{tabular}{crrr}
\hline Parâmetros & Estimativas & Erro Padrão & p-valor \\
\hline$\gamma_{00}$ & 83,412 & 1,003 & $<0,0001$ \\
$\gamma_{10}$ & 0,582 & 0,130 & $<0,0001$ \\
$\beta_{2}$ & 1,131 & 1,380 & 0,4137 \\
$\beta_{3}$ & 1,102 & 1,435 & 0,4437 \\
$\beta_{4}$ & $-0,440$ & 1,276 & 0,7307 \\
$\tau_{00}$ & 44,668 & 8,087 & $<0,0001$ \\
$\tau_{11}$ & 0,037 & 0,198 & 0,8518 \\
$\tau_{01}$ & 1,475 & 0,725 & 0,0419 \\
$\sigma^{2}$ & 100,378 & 4,539 & $<0,0001$ \\
Estatística & $-2^{*} \log$-veros. & 8691,148 & - \\
\hline
\end{tabular}

Com isso, o modelo final a ser considerado para a variável pads ${ }_{i j}$ deve levar em consideração apenas a variável explanatória IMCC, ou seja, o modelo dado em (17).

A Figura 6 representa graficamente o valor estimado da variável pads ${ }_{i j}$, de acordo com o modelo (17), na qual observa-se também diferenças nos interceptos e igualdade nas inclinações. 


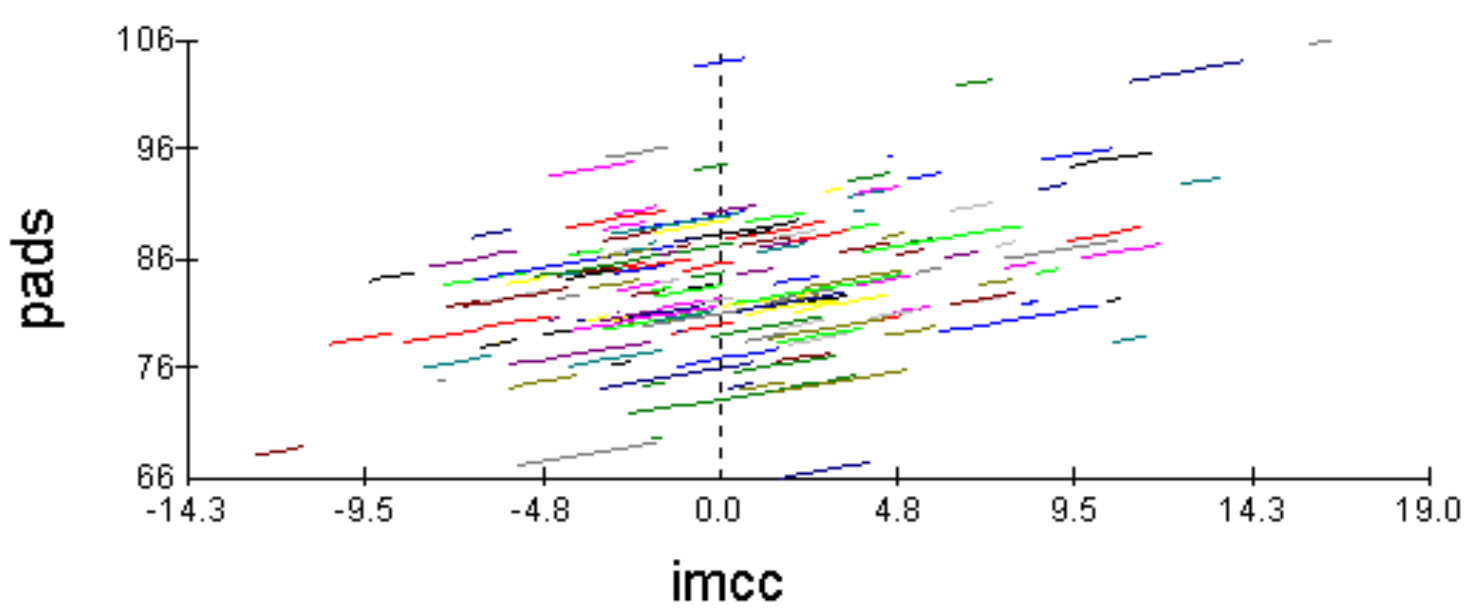

Figura 6 - Gráfico do modelo final para a variável $\operatorname{pads}_{i j}$

Para a variável pressão arterial sistólica em pé, $\operatorname{pasp}_{i j}$, com o modelo (16), tem-se que as estimativas dos parâmetros são mostradas na Tabela 10.

Tabela 10. Estimativa dos parâmetros do modelo (16) considerando apenas os interceptos para os dados de pressão arterial sistólica em pé - "PAI" .

\begin{tabular}{ccrr}
\hline Parâmetros & Estimativas & Erro Padrão & p-valor \\
\hline$\gamma_{00}$ & 151,917 & 3,737 & $<0,0001$ \\
$\tau_{00}$ & 496,640 & 141,179 & 0,0004 \\
$\sigma^{2}$ & 355,585 & 66,160 & $<0,0001$ \\
Estatística & $-2^{*}$ log-veros. & 988,297 & - \\
\hline
\end{tabular}

A média da pressão arterial sistólica em pé dos idosos é dada por 151,917 mmHg. Para os resíduos, a maior variação, neste caso, está entre os idosos $\left(\tau_{00}=496,640\right)$ do que entre ocasiões dentro dos idosos $\left(\sigma^{2}=355,585\right)$, indicando uma maior variação na pressão arterial sistólica em pé dentro dos idosos.

Neste caso, 58,28\% da variabilidade está dentro dos idosos, ou seja,

$$
\hat{\rho}=\frac{496,640}{496,640+355,585}=0,5828
$$


Incluindo-se a variável explanatória IMCC ao modelo (16), tem-se as estimativas dos parâmetros considerados mostrados na Tabela 11, através do modelo (17).

Tabela 11. Estimativa dos parâmetros do modelo (17) considerando IMCC como uma variável explanatória para os dados de pressão arterial sistólica em pé "PAI"

\begin{tabular}{crrr}
\hline Parâmetros & Estimativas & Erro Padrão & p-valor \\
\hline$\gamma_{00}$ & 151,703 & 3,796 & $<0,0001$ \\
$\gamma_{01}$ & 1,052 & 0,720 & 0,1497 \\
$\tau_{00}$ & 470,630 & 139,074 & 0,0007 \\
$\sigma^{2}$ & 363,811 & 68,358 & $<0,0001$ \\
Estatística & $-2^{*}$ log-veros. & 958,711 & - \\
\hline
\end{tabular}

Neste caso, o intercepto de 151,703 mmHg representa a pressão arterial sistólica em pé esperada no j-ésimo idoso quando o IMCC for igual a 25,2482 (valor médio $M$ da variável). Há um aumento da pressão arterial sistólica em pé de 1,052 mmHg quando o IMCC aumenta de uma unidade em relação à média geral 25,2482. Pouca alteração novamente ocorre para os resíduos em relação ao modelo considerando apenas os interceptos, isto é, entre os idosos $\left(\tau_{00}=470,630\right)$ e entre ocasiões dentro dos idosos $\left(\sigma^{2}=363,811\right)$, continuando com uma variação significativa $(\mathrm{p}$-valor $=0,0007)$ entre os idosos do que entre ocasiões dentro dos idosos, mesmo após a inclusão da variável IMCC.

A correlação intra-idosos é dada por:

$$
\hat{\rho}=\frac{470,630}{470,630+363,811}=0,5640,
$$

que praticamente não muda em relação ao modelo anterior.

A estatística -2 vezes o logaritmo da função de verossimilhança para esse modelo é igual a 958,711, considerando, neste caso, apenas 103 valores do total de 
3652 observações. Há uma redução significativa $(988,297$ - 958,711 = 29,586) do modelo com a variável IMCC em relação ao modelo só com os interceptos, comparando-se como quantil de uma distribuição $\chi^{2}$ com 1 grau de liberdade $(p<0,0001)$. Portanto, há uma variação da pressão arterial de idoso para idoso para diferentes valores do IMCC.

De acordo com o modelo (18), adicionando-se, agora, a variável IMCC com efeito aleatório para o nível dos idosos, as estimativas dos parâmetros são mostradas na Tabela 12 .

Tabela 12. Estimativa dos parâmetros do modelo (18) considerando o IMCC com efeito aleatório para o nível dos idosos para os dados de pressão arterial sistólica em pé - "PAI" .

\begin{tabular}{crrr}
\hline Parâmetros & Estimativas & Erro Padrão & p-valor \\
\hline$\gamma_{00}$ & 151,966 & 3,561 & $<0,0001$ \\
$\gamma_{10}$ & 0,204 & 0,876 & 0,8169 \\
$\tau_{00}$ & 318,680 & 137,017 & 0,0200 \\
$\tau_{11}$ & 5,424 & 5,363 & 0,3118 \\
$\tau_{01}$ & 7,740 & 17,648 & 0,6696 \\
$\sigma^{2}$ & 363,157 & 68,336 & $<0,0001$ \\
Estatística & $-2 * \log$-veros. & 956,035 & - \\
\hline
\end{tabular}

Da Tabela 12, o valor de 151,966 mmHg representa a média da pressão arterial sistólica em pé dos idosos controlada pelo IMCC das ocasiões. A inclinação média, representando a relação entre essa pressão e o IMCC das ocasiões, é dada por 0,204 . 
A matriz de covariância é:

$$
\left(\begin{array}{cc}
\hat{\tau}_{00} & \hat{\tau}_{01} \\
\hat{\tau}_{10} & \hat{\tau}_{11}
\end{array}\right)=\left(\begin{array}{cc}
318,680 & 7,740 \\
7,740 & 5,424
\end{array}\right) .
$$

Neste caso, a covariância entre os interceptos e as inclinações é dada por 7,740 , pequena em relação ao seu erro padrão $(17,648)$, sendo, portanto, não significativa ( $\mathrm{p}$-valor $=0,6696$ ), indicando uma ausência dos efeitos dos IMCC's das ocasiões sobre os diferentes valores de pressão arterial diastólica em pé no idoso. Já o valor 5,424, representando a variação nas inclinações, também possui um erro padrão alto $(5,363)$, sendo, também, não significativo ( $\mathrm{p}$-valor $=0,3118)$, indicando a não ocorrência de variabilidade nos interceptos. No entanto, o valor 318,680 representa uma variabilidade significativa nos interceptos, ou seja, as pressões arteriais diastólica em pé dos idosos continuam diferindo, em média, mesmo após a inclusão do efeito da variável IMCC.

Considerando os 103 valores do total de 3652 observações, o valor da estatística -2 vezes o logaritmo da função de verossimilhança foi de 956,035 o que leva novamente a uma redução não significativa do modelo com a variável IMCC com efeito aleatório para o nível dos idosos em relação ao modelo considerando a IMCC como uma variável explanatória $(958,711$ - 956,035 =2,676), se comparada com o quantil de uma distribuição $\chi^{2}$ com 2 graus de liberdade ( $\mathrm{p}$-valor $=0,2624$ ), o que mostra a não existência de uma variação da pressão arterial sistólica sentado de idoso para idoso através das ocasiões.

Incluindo as variáveis explanatórias sexo, raça e estado civil do segundo nível ao modelo, tanto no MLwiN como no SAS não houve convergência no método de ajuste. As estimativas dos parâmetros para o modelo (19), com a retirada a variável explanatória sexo $\beta_{2}$, para uma convergência no método de ajuste, são apresentadas na Tabela 13. 
Tabela 13. Estimativa dos parâmetros do modelo (19) considerando o IMCC com efeito aleatório para o nível dos idosos mais as variáveis explanatórias desse nível para os dados de pressão arterial sistólica em pé - "PAI" .

\begin{tabular}{crrr}
\hline Parâmetros & Estimativas & Erro Padrão & p-valor \\
\hline$\gamma_{00}$ & 150,509 & 4,813 & $<0,0001$ \\
$\gamma_{10}$ & 0,388 & 0,884 & 0,6625 \\
$\beta_{3}$ & 0,404 & 9,344 & 0,9657 \\
$\beta_{4}$ & 3,378 & 7,909 & 0,6714 \\
$\tau_{00}$ & 357,061 & 144,950 & 0,0138 \\
$\tau_{11}$ & 4,435 & 5,135 & 0,3878 \\
$\tau_{01}$ & 9,449 & 17,541 & 0,5901 \\
$\sigma^{2}$ & 364,263 & 68,536 & $<0,0001$ \\
Estatística & $-2^{*} \log$-veros. & 955,905 & - \\
\hline
\end{tabular}

Verifica-se, analisando a Tabela 13, que houve pouca alteração nas estimativas dos parâmetros. Portanto, mesmo após a inclusão das variáveis explanatórias raça e estado civil para o nível dos idosos, através do modelo (19), não há alteração nas conclusões obtidas para a variável resposta $\operatorname{pasp}_{i j}$. Isto é comprovado através da diferença entre as estatísticas -2 vezes o logaritmo da função de verossimilhança $(956,035$ - 955,905 =0,130), pois a redução nessa estatística não é significativa, se comparada com o quantil de uma distribuição $\chi^{2}$ com 2 graus de liberdade (p-valor $=0,9371)$.

Assim sendo, o modelo final a ser considerado para a variável pasp p $_{i j}$, leva em consideração apenas a variável explanatória IMCC, ou seja, o modelo dado em (17).

O resultado apresentado graficamente (Figura 7) representa o valor estimado da variável $\operatorname{pasp}_{i j}$, de acordo com o modelo (17), observando-se diferenças 
nos interceptos e igualdade nas inclinações.

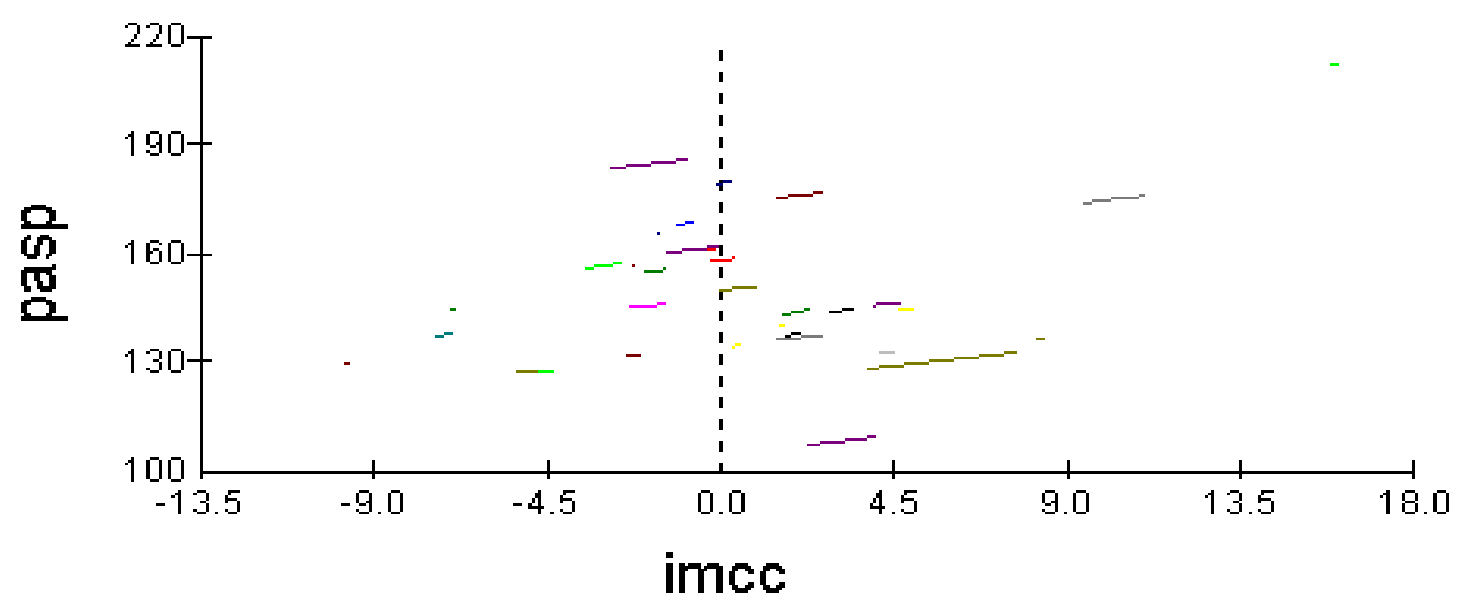

Figura 7 - Gráfico do modelo final para a variável pasp $p_{i j}$

Para a variável pressão arterial diastólica em pé, padp ${ }_{i j}$, seguindo o modelo dado em (16), as estimativas dos parâmetros são dadas na Tabela 14.

Tabela 14. Estimativa dos parâmetros do modelo (16) considerando apenas os interceptos para os dados de pressão arterial diastólica em pé - "PAI" .

\begin{tabular}{crrc}
\hline Parâmetros & Estimativas & Erro Padrão & p-valor \\
\hline$\gamma_{00}$ & 90,329 & 1,824 & $<0,0001$ \\
$\tau_{00}$ & 106,651 & 33,525 & $<0,0015$ \\
$\sigma^{2}$ & 101,608 & 19,044 & $<0,0001$ \\
Estatística & $-2^{*}$ log-veros. & 829,706 & - \\
\hline
\end{tabular}

Os valores da Tabela 14 mostram uma estimativa de 90,329 mmHg para a média da pressão arterial diastólica em pé dos idosos. Já os resíduos, considerados como efeitos aleatórios, são iguais a $\tau_{00}=106,651$ e $\sigma^{2}=101,608$, o que mostra uma variabilidade significativa semelhante entre os idosos e entre ocasiões dentro 
dos idosos em relação à variação total $(106,651+101,608=208,259)$. Para verificar essa variabilidade, nos idosos, pode-se estimar a correlação intra-idosos:

$$
\hat{\rho}=\frac{106,651}{106,651+101,608}=0,5119,
$$

ou seja, 51,19\% de variabilidade intra-idosos.

Considerando o modelo (17), tem-se as estimativas dos parâmetros, mostrados na Tabela 15, quando da inclusão da variável explanatória IMCC ao modelo (16).

Tabela 15. Estimativa dos parâmetros do modelo (17) considerando IMCC como uma variável explanatória para os dados de pressão arterial diastólica em pé - "PAI" .

\begin{tabular}{crrr}
\hline Parâmetros & Estimativas & Erro Padrão & p-valor \\
\hline$\gamma_{00}$ & 89,541 & 1,839 & $<0,0001$ \\
$\gamma_{01}$ & 0,666 & 0,345 & 0,0588 \\
$\tau_{00}$ & 99,533 & 32,506 & 0,0022 \\
$\sigma^{2}$ & 101,408 & 19,220 & $<0,0001$ \\
Estatística & $-2^{*} \log$-veros. & 802,235 & - \\
\hline
\end{tabular}

O valor de 89,541 mmHg na Tabela 15, representa um valor médio para o intercepto, ou seja, a pressão arterial diastólica em pé esperada no j-ésimo idoso quando o valor da variável IMCC for igual a 25,2482 (valor médio $M$ para a variável). Já a estimativa positiva para a variável $\operatorname{IMCC}(0,666)$, indica um aumento da pressão arterial diastólica em pé com o aumento do IMCC, ou seja a pressão arterial diastólica em pé aumenta de 0,666 mmHg, quando o IMCC aumenta de uma unidade em relação à média geral 25,2482. No entanto, o efeito dessa variável no modelo apresenta um p-valor de 0,0588, o que será considerado significativo a 5\% de probabilidade, ou seja, será considerado que existe um efeito nas inclinações para cada idoso. Para os efeitos aleatórios (resíduos), pouca alteração é verificada em relação ao modelo apenas com 
os interceptos, ou seja, entre os idosos $\left(\tau_{00}=99,533\right)$ e entre ocasiões dentro dos idosos $\left(\sigma^{2}=101,408\right)$, apesar de continuarem sendo significativos, indicando uma influência das ocasiões na pressão arterial diastólica em pé para cada idoso, mesmo após a inclusão da variável IMCC.

A correlação intra-idosos é dada por:

$$
\hat{\rho}=\frac{99,533}{99,533+101,408}=0,4953
$$

que praticamente não muda em relação ao modelo anterior.

Considerando os 104 valores do total de 3652 observações a estatística -2 vezes o logaritmo da função de verossimilhança para esse modelo é igual a 802,235 indicando uma redução significativa $(829,706$ - 802,235 = 27,471) em relação ao modelo anterior, comparada com o quantil de uma distribuição $\chi^{2}$ com 1 grau de liberdade $(p<0,0001)$ Assim, há uma variação da pressão arterial de idoso para idoso com diferentes IMCC.

Considerando-se o modelo (18), em que a variável IMCC é colocada como efeito aleatório para o nível dos idosos, ou seja, não apenas a relação entre os IMCC's pode variar através dos idosos, mas também a pressão em uma determinada ocasião está relacionada com um IMCC dessa ocasião. A estimativas dos parâmetros são mostradas na Tabela 16.

Neste caso, tanto no MLwiN como no SAS, a matriz de covariâncias, não é positiva definida, assim sendo, os valores de $\tau_{11}$ e $\tau_{01}$ não são estimados. Desse modo, não há como adicionar as variáveis explanatórias sexo, raça e estado civil do segundo nível. Novamente o modelo final a ser considerado para a variável padp $i j$ leva em consideração apenas a variável explanatória IMCC, ou seja, o modelo (17).

O resultado apresentado graficamente (Figura 8) representa o valor estimado da variável padp ${ }_{i j}$, de acordo com o modelo (17), observando-se diferenças nos interceptos e igualdade nas inclinações. 
Tabela 16. Estimativa dos parâmetros do modelo (18) considerando o IMCC como efeito aleatório para o nível dos idosos para os dados de pressão arterial diastólica em pé - "PAI" .

\begin{tabular}{crrr}
\hline Parâmetros & Estimativas & Erro Padrão & p-valor \\
\hline$\gamma_{00}$ & 89,541 & 1,839 & $<0,0001$ \\
$\gamma_{10}$ & 0,666 & 0,345 & 0,0588 \\
$\tau_{00}$ & 99,460 & 32,557 & 0,0023 \\
$\tau_{11}$ & 0,000 & 0,000 & - \\
$\tau_{01}$ & 0,000 & 0,000 & - \\
$\sigma^{2}$ & 101,446 & 19,158 & $<0,0001$ \\
Estatística & $-2^{*} \log$-veros. & 802,234 & - \\
\hline
\end{tabular}

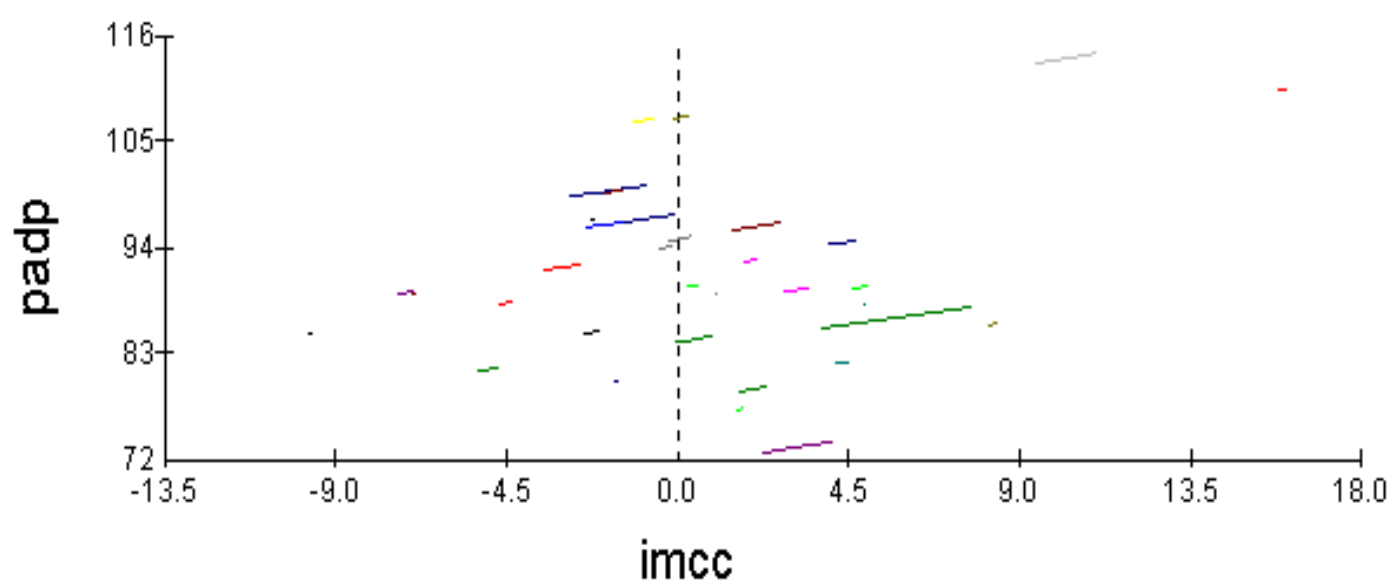

Figura 8 - Gráfico do modelo final para a variável padp ${ }_{i j}$ 


\subsection{Resultados da análise dos dados do teor de proteína no leite}

Para os dados de proteína no leite, apresentado em Diggle (1994), o modelo a ser utilizado, também com dois níveis, tem neste caso, as observações dos teores de proteína no leite alocados no nível 1 e os animais no nível 2. Assim, os dados representam vários teores de proteína para um mesmo animal. Neste caso, as variáveis consideradas são:

Variáveis do nível 1 (observações)

- teor $i_{i j}$ : valor do teor de proteína no leite na $i$-ésima observação para o $j$-ésimo animal;

- tempo ${ }_{i j}$ : número de semanas após o primeiro parto na $i$-ésima observação para j-ésimo animal;

Variável do nível 2 (animais)

- trat $_{j}= \begin{cases}0 & \text { se o animal recebeu a dieta de cevada } \\ 1 & \text { se o animal recebeu a mistura de cevada e tremoço } \\ 2 & \text { se o animal recebeu a dieta só de tremoço }\end{cases}$

Considerando a variável resposta como o teor de proteína no leite, teor $_{i j}$, e seguindo o modelo análogo ao dado em (16), as estimativas dos parâmetros considerados são mostradas na Tabela 17.

De acordo com os resultados da Tabela 17, tem-se apenas o intercepto como um efeito fixo, cujo valor da estimativa é de 3,424, representando o teor médio de proteína no leite dos animais. Numa análise dos resíduos, considerados como aleatórios, tem-se uma maior variação entre as observações dentro dos animais $\left(\sigma^{2}\right.$ $=0,076)$ do que apenas entre os animais $\left(\tau_{00}=0,034\right)$, indicando uma influência das observações no teor de proteína no leite para cada animal. 
Tabela 17. Estimativa dos parâmetros do modelo análogo ao (16) para os dados de teor de proteína do leite, considerando apenas os interceptos.

\begin{tabular}{ccrc}
\hline Parâmetros & Estimativas & Erro Padrão & p-valor \\
\hline$\gamma_{00}$ & 3,424 & 0,022 & $<0,0001$ \\
$\tau_{00}$ & 0,034 & 0,006 & $<0,0001$ \\
$\sigma^{2}$ & 0,076 & 0,003 & $<0,0001$ \\
Estatística & $-2^{*}$ log-veros. & 513,141 & - \\
\hline
\end{tabular}

O valor da correlação intra-animais é dado por:

$$
\hat{\rho}=\frac{0,034}{0,034+0,076}=0,3091
$$

ou seja, 30,91\% de variabilidade intra-animais.

Adicionando-se a seguir, a variável TEMPO ao modelo como uma variável explanatória e, seguindo agora o modelo análogo ao dado em (17), temse as estimativas dos parâmetros mostrados na Tabela 18.

Tabela 18. Estimativa dos parâmetros do modelo análogo ao (17) considerando TEMPO como uma variável explanatória para os dados de teor de proteína do leite.

\begin{tabular}{crrc}
\hline Parâmetros & Estimativas & Erro Padrão & p-valor \\
\hline$\gamma_{00}$ & 3,480 & 0,026 & $<0,0001$ \\
$\gamma_{01}$ & $-0,006$ & 0,001 & $<0,0001$ \\
$\tau_{00}$ & 0,034 & 0,006 & $<0,0001$ \\
$\sigma^{2}$ & 0,075 & 0,003 & $<0,0001$ \\
Estatística & $-2 * \log$-veros. & 495,765 & - \\
\hline
\end{tabular}


Analisando a Tabela 18, tem-se o intercepto igual a 3,480, representando o teor de proteína no leite no $j$-ésimo animal quando o TEMPo for igual a zero. A estimativa negativa para a variável TEMPo (-0,006), indica uma diminuição do teor de proteína no leite com o aumento de uma semana no TEMPO. Para os efeitos aleatórios (resíduos), observa-se pouca alteração, em relação ao modelo apenas com os interceptos, ou seja, entre observações dentro dos animais $\left(\sigma^{2}=0,075\right)$ e entre os animais $\left(\tau_{00}=0,034\right)$, indicando uma influência das observações no teor de proteína do leite para cada animal. Nota-se também que, mesmo após a inclusão da variável TEMPO, com a correlação intra-animais praticamente igual (correlação intra-animal $=0,3119)$.

A estatística -2 vezes o logaritmo da função de verossimilhança para esse modelo é igual a 495,7651, considerando 1337 valores do total de 1501 observações, mostrando uma redução significativa $(513,141-495,765=17,376)$ do modelo com a variável TEMPO em relação ao modelo apenas com os interceptos, considerando-se uma distribuição $\chi^{2}$ com 1 grau de liberdade ( $\mathrm{p}$-valor $=0,0002$ ) Assim, verifica-se uma variação no teor de proteína do leite de animal para animal nos diferentes TEMPOS.

Incluindo, agora, o TEMPO com efeito aleatório para o nível dos animais, ou seja, não apenas o teor de proteína no leite em uma determinada observação está relacionada com o TEMPO, mas também a relação entre os TEMPOS pode variar através dos animais, tem-se, considerando o modelo análogo ao (18), as estimativas dadas pela Tabela 19 .

Da Tabela 19, o teor de proteína no leite controlado pelo TEMPo é de 3,517 e o valor -0,013 é a inclinação média representando a relação entre esse teor de proteína e o TEMPO.

A matriz de covariância estimada é:

$$
\left(\begin{array}{ll}
\hat{\tau}_{00} & \hat{\tau}_{01} \\
\hat{\tau}_{10} & \hat{\tau}_{11}
\end{array}\right)=\left(\begin{array}{cc}
0,073 & -0,005 \\
-0,005 & 0,001
\end{array}\right)
$$


Tabela 19. Estimativa dos parâmetros do modelo análogo ao (18) considerando o TEMPO com efeito aleatório para o nível dos animais para os dados de teor de proteína no leite.

\begin{tabular}{crrr}
\hline Parâmetros & Estimativas & Erro Padrão & p-valor \\
\hline$\gamma_{00}$ & 3,517 & 0,033 & $<0,0001$ \\
$\gamma_{10}$ & $-0,013$ & 0,003 & $<0,0001$ \\
$\tau_{00}$ & 0,073 & 0,014 & $<0,0001$ \\
$\tau_{11}$ & 0,001 & 0,000 & $<0,0001$ \\
$\tau_{01}$ & $-0,005$ & 0,001 & $<0,0001$ \\
$\sigma^{2}$ & 0,060 & 0,002 & $<0,0001$ \\
Estatística & $-2^{*} \log$-veros. & 356,738 & - \\
\hline
\end{tabular}

A variação nos interceptos é dada por 0,073, enquanto que 0,001 representa a variação nas inclinações. Já, a covariância entre os interceptos e as inclinações é dada por -0,005. Observa-se, assim, uma variabilidade nos interceptos, ou seja, os teores de proteína no leite, mesmo após a inclusão do efeito do TEMPO, diferem, em média; uma variabilidade nas inclinações, apresentada por um erro padrão de 0,0001 para uma estimativa do parâmetro igual a 0,001 $(p<0,0001)$. Além disso, nota-se que a covariância negativa entre os interceptos e as inclinações (-0,005) com um erro padrão de 0,001 $(p<0,0001)$, indicando uma relação negativa entre eles. Desse modo, há o efeito dos TEMPOs sobre os diferentes valores do teor de proteína no leite para os animais.

O valor da estatística -2 vezes o logaritmo da função de verossimilhança foi 356,738, nos mesmos 1337 valores do total de 1501 observações, mostrando uma redução significativa do modelo com a variável TEMPO com efeito aleatório para o nível dos animais e o modelo considerando a variável TEMPO como uma variável explanatória fixa $(495,765$ - 356,738 = 139,027), se comparado a uma distribuição $\chi^{2}$ com 2 graus de liberdade $(p<0,0001)$. Assim, tem-se uma melhora no modelo 
quando se considera as inclinações como efeitos aleatórios, indicando, portanto, que há uma variação do teor de proteína no leite de animal para animal.

Incluindo agora, a variável explanatória do segundo nível, a dieta, e seguindo o modelo (20), as estimativas dos parâmetros são fornecidas na Tabela 20.

Tabela 20. Estimativa dos parâmetros do modelo (20) considerando o TEMPO com efeito aleatório para o nível dos animais e a variável explanatória dieta para os dados de teor de proteína no leite.

\begin{tabular}{crrr}
\hline Parâmetros & Estimativas & Erro Padrão & p-valor \\
\hline$\gamma_{00}$ & 3,617 & 0,045 & $<0,0001$ \\
$\gamma_{10}$ & $-0,013$ & 0,003 & 0,1339 \\
$\beta_{2}$ & $-0,094$ & 0,050 & 0,0639 \\
$\beta_{3}$ & $-0,197$ & 0,050 & 0,0002 \\
$\tau_{00}$ & 0,072 & 0,014 & $<0,0001$ \\
$\tau_{11}$ & 0,001 & 0,000 & $<0,0001$ \\
$\tau_{01}$ & $-0,005$ & 0,001 & $<0,0001$ \\
$\sigma^{2}$ & 0,060 & 0,002 & $<0,0001$ \\
Estatística & $-2^{*} \log$-veros. & 342,184 & - \\
\hline
\end{tabular}

Ao acrescentar-se a variável dieta observa-se uma redução significativa ( $\mathrm{p}$-valor $=0,0002)$ no teor de proteína do leite para a dieta de tremoço $(-0,197)$ e próximo a significância ( $\mathrm{p}$-valor $=0,0639)$ para a mistura $(-0,094)$. Há uma diferença $(356,738-342,184=14,554)$ significativa $(p<0,0001)$ nos valores da estatística -2 vezes o logaritmo da função de verossimilhança, se compararmos o valor da diferença com o quantil de uma distribuição $\chi^{2}$ com 2 graus de liberdade, em relação a um modelo análogo ao (18) com a variável explanatória TEMPO com efeito aleatório para o nível dos animais, indicando uma diminuição do teor de proteína no leite de animal para animal após a inclusão da variável explanatória dieta de tremoço e a mistura (cevada e tremoço) em relação à dieta de cevada. 
Na Tabela 21, encontra-se as estimativas dos parâmetros, seguindo o modelo (21), em que as interações das dietas com o tempo foram acrescentadas ao modelo (20)

Tabela 21. Estimativa dos parâmetros do modelo (21) considerando o TEMPO com efeito aleatório para o nível dos animais e suas interações com a variável explanatória desse nível para os dados de teor de proteína no leite.

\begin{tabular}{crrr}
\hline Parâmetros & Estimativas & Erro Padrão & p-valor \\
\hline$\gamma_{00}$ & 3,588 & 0,059 & $<0,0001$ \\
$\gamma_{10}$ & $-0,009$ & 0,006 & 0,1339 \\
$\beta_{2}$ & $-0,083$ & 0,082 & 0,3147 \\
$\beta_{3}$ & $-0,124$ & 0,082 & 0,1347 \\
$\beta_{4}$ & $-0,009$ & 0,008 & 0,2608 \\
$\beta_{5}$ & $-0,001$ & 0,008 & 0,9005 \\
$\tau_{00}$ & 0,073 & 0,014 & $<0,0001$ \\
$\tau_{11}$ & 0,001 & 0,000 & $<0,0001$ \\
$\tau_{01}$ & $-0,005$ & 0,001 & $<0,0001$ \\
$\sigma^{2}$ & 0,060 & 0,002 & $<0,0001$ \\
Estatística & $-2^{*} \log$-veros. & 340,707 & - \\
\hline
\end{tabular}

Analisando a Tabela 21, verifica-se que os valores das estimativas dos parâmetros fixos são todos não significativos $(p>0,05)$ com a inclusão das interações das dietas com o tempo. Portanto, não há uma interação das dietas com o tempo, considerando uma relação linear nos parâmetros. A exceção é o intercepto $(p<$ 0,001), indicando uma variabilidade de animal para animal no teor de proteína do leite, mesmo após o acréscimo das interações. A diferença $(342,184-340,707=$ 1,477) nos valores da estatística -2 vezes o logaritmo da função de verossimilhança é não significativa ( $\mathrm{p}$-valor $=0,4748$ ) para os modelos considerados, se compararmos o valor da diferença com o quantil de uma distribuição $\chi^{2}$ com 2 graus de liberdade. 
Assim, a relação no teor de proteína do leite com o tempo e as dietas é melhor representado através do modelo (20).

O resultado apresentado graficamente (Figura 9) representa o valor estimado da variável teor ${ }_{i j}$ de acordo com o modelo (20), observando-se diferenças nos interceptos e igualdade nas inclinações, mostrando a redução no teor de proteína mesmo após a inclusão das dietas.

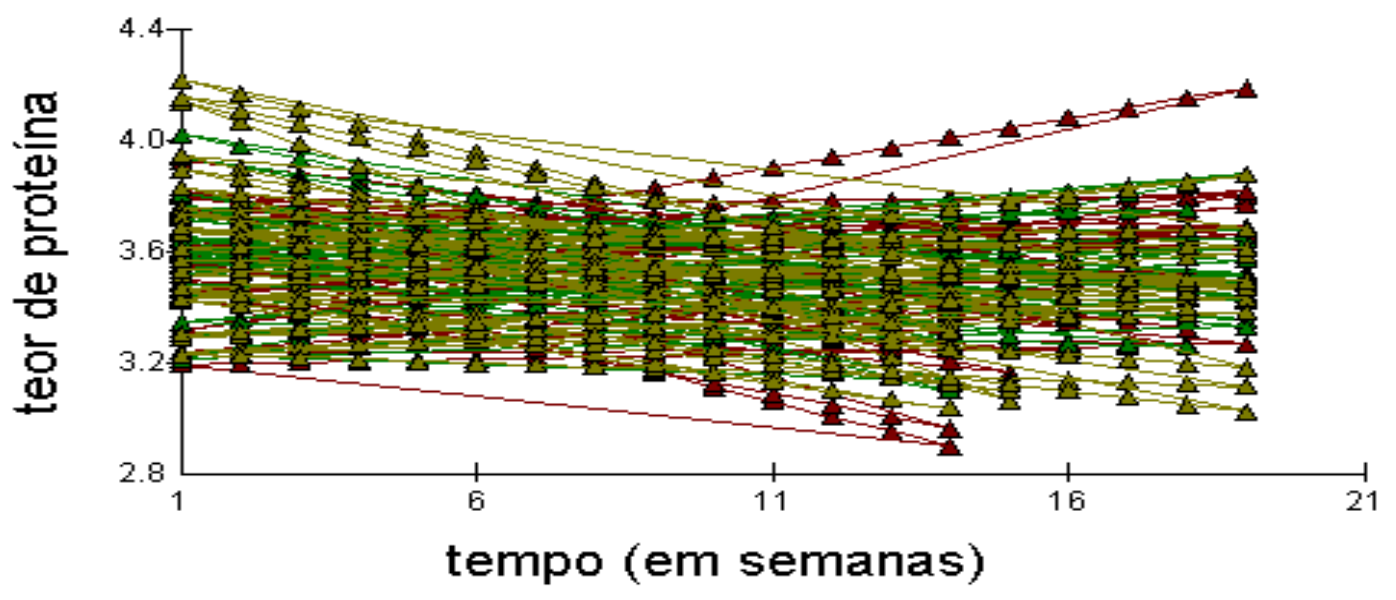

Figura 9 - Gráfico do modelo final para a variável teor ${ }_{i j}$ 


\section{CONCLUSÕES}

De acordo com os objetivos deste estudo, pode-se concluir que:

- quando os dados estão organizados segundo uma estrutura hierárquica, é importante levar em conta essa estrutura, uma vez, que não fazê-la pode implicar na superestimação dos coeficientes do modelo em estudo;

- quando se tem dados de medidas repetidas no tempo, é interessante considerar uma estrutura hierárquica para esses dados, já que é possível verificar as possíveis correlações existentes em cada nível da estrutra com as possíveis variações dos tempos em cada indivíduo;

- o software MLwiN mostrou-se muito útil no ajuste dos modelos hierárquicos, mostrando uma interatividade grande com o usuário, podendo ser obtido gráficos para exploração do modelo, análise de resíduos e uma modelagem da variância em função das variáveis explanatórias;

- esse software também se mostrou útil na presença de grandes conjuntos de dados e eficiente na estimação de parâmetros, apresentando alguns problemas na ausência de um grande número de informações nas diferentes ocasiões de medida;

- já o software SAS através do PROC MIXED, além da eficiência na estimação dos parâmetros envolvidos no modelo, apresentando também problemas na presença de observações faltantes nas diferentes ocasiões de medida, fornece diretamente os níveis de significância para os testes dos parâmetros estimados, o que não ocorre no MLwiN; 
- para os dados do "PAI" , levar em conta a estrutura hierárquica dos dados revelou que as diferentes medidas de pressão arterial estão relacionadas positivamente com o IMC ao longo do tempo e, revelou também que a variação na pressão arterial não depende de sexo, raça e estado civil;

- para os dados do teor de proteína no leite, verificou-se haver uma redução do teor de proteína no leite ao longo do tempo, mesmo com a inclusão das dietas, fato este que foi independente da interação do tempo com o tipo de dieta ;

Como uma continuidade deste estudo, seria interessante utilizar a estrutura hierárquica aplicada a medidas repetidas no tempo considerando variáveis que possuem uma distribuição discreta (binária, binomial ou Poisson) e modelos com variáveis cujos coeficientes podem ser não lineares. 


\section{REFERÊNCIAS BIBLIOGRÁFICAS}

AITKIN, M.; LONGFORD, N. Statistical modelling in school effectiveness studies. Journal of the Royal Statistical Society. Series A, v.149, p.1-43, 1986.

AITKIN, M.; ANDERSON, D.; HINDE, J. Statistical modelling of data on teaching styles. Journal of the Royal Statistical Society. Series A, v.144, p.148-161, 1981.

BENNETT, N. Teaching styles and pupil progress. Cambrige: Harvard University Press, 1976. 201p.

BRYK, A.S.; RAUDENBUSH, S.W. Hierarchical linear models. Newbury Park: Sage, 1992. 202p.

BRYK,A.S.; RAUdenBush, S.W.; SELtZER, M.; CONGDON, R. An introduction to HLM: computer program and user's guide. 2.ed. Chicago: University of Chicago, Dept. of Education, 1988. 120p.

DIGGLE, P.J.; LIANG, K.Y.; ZEGER, S.L. Analysis of longitudinal data. New York: Oxford University Press, 1994. 253p.

GOLDSTEIN, H. Multilevel mixed linear model analysis using interative generalized least square. Biometrika, v.73, n.1, p.43-56, 1986.

GOLDSTEIN, H. Multilevel statistical models. 2.ed. London: Edward Arnold, 1995. 192p. 
HARVILLE, D.A. Maximum likelihood approaches to variance component estimation and to related problems. Journal of the American Statistical Association, v.72, p.320-337, 1977.

KREFT, I.G.; DE LEEUW, J.; VAN DER LEEDEN, R. Review of five multilevel analysis programs: BMDP-5V, GENMOD, HLM, ML3, and VARCL. The American Statistician, v.48, n.4, p.324-335, 1994.

LitTEll, R.C.; MILlikEN, G.A.; STROUP, W.W. et al. SAS system for mixed models. Cary: SAS Institute, 1996. 633p.

LONGFORD, N.T. VARCL: software for variance component analysis of data with hierarchically nested random effects (maximum likelihood). Princeton: Educational Testing Service, 1988. 149p.

LONGFORD, N.T. Random coefficient models. Oxford: Clarendon Press, 1993. $270 \mathrm{p}$.

MASON, W.M.; WONG, G.Y.; ENTWISLE, B. Contextual analysis through the multilevel linear model. Sociological Methodology, p.72-103, 1984.

MASON, W.M.; ANDERSON, A.F.; HAYAT, N. Manual for GENMOD. Ann Arbor: University of Michigan Population Studies Center, 1988. 170p.

NETER, J.; WASSERMAN, W.; KUTNER, M.H. Applied linear statistical models: regression, analysis of variance and experimental designs. 3.ed. Burr Ridge: R.D. Irwin, 1990. 1181p.

RASBASH, J.; BROWNE, W.; GOLDSTEIN, H. et al. A user's guide to MLwiN. London: s.ed., 2001. 278p.

SEARLE, S.R.; CASELlA, G.; MCCULLOCH, C.E. Variance components. New York: John Wiley, 1992. 501p. 
SINGER, J.D. Using SAS proc mixed to fit multilevel models, hierarchical models, and individual growth models. Journal of Educational and Behavioral Statistics, v.24, n.4, p.323-355, 1998.

SINGER, J.M. ANDRADE, D.F. de Análise de dados longitudinais. Campinas: ABE, 1986. 106p.

SMITH, D.M. Oswald: object-oriented software for the analysis of longitudinal data in S. http://www.maths.lancs.ac.uk./Software/Oswald. (Nov. 1997).

SULLIVAN, L.M.; DUKES, K.; LOSINA, E. Tutorial in biostatistics an introduction to hierarchical linear modelling. Statistics in Medicine, v.18, p.855-888, 1999.

ZHOU, X.; PERKINS, A.J.; HUI, S.L. Comparisons of software packages for generalized linear multilevel models. The American Statistician, v.53, n.3, p.282-290, 1999. 


\section{APÊNDICES}




\title{
APÊNDICE 1
}

\author{
Programas SAS
}


Programas SAS para os dados do "PAI" com a variável explanatória pressão arterial sistólica sentado.

- Programa para o modelo (16), considerando apenas os interceptos

proc mixed noclprint covtest;

class idoso ;

model pass $=/$ solution ;

random intercept /subject=idoso;

run;

- Programa para o modelo (17), considerando IMCC como uma variável explanatória

proc mixed noclprint covtest;

class idoso ;

model pass =imcc/solution ddfm=bw;

random intercept /subject=idoso;

run;

. Programa para o modelo (18), considerando o IMCC com efeito aleatório para o nível dos idosos

proc mixed noclprint covtest;

class idoso ;

model pass =imcc / solution ddfm=bw;

random intercept imcc/subject=idoso type=un;

run;

. Programa para o modelo (19), considerando o IMCC com efeito aleatório para o nível dos idosos mais as variáveis explanatórias desse nível

proc mixed noclprint covtest;

class idoso sexo estciv cor;

model pass =imcc sexo estciv cor / solution ddfm=bw;

random intercept imcc/subject=idoso type=un;

run; 
Programas SAS para os dados do teor de proteína no leite.

. Programa para o modelo análogo ao (16), considerando apenas os interceptos

proc mixed noclprint covtest;

class animal dieta;

model teor $=/$ solution $\mathrm{ddfm}=\mathrm{bw}$;

random intercept /subject=animal type=un;

run;

. Programa para o modelo análogo ao (17), considerando TEMPo como uma variável explanatória

proc mixed noclprint covtest;

class animal dieta;

model teor $=$ tempo /solution ddfm=bw;

random intercept /subject=animal type=un;

run;

. Programa para o modelo análogo ao (18), considerando o TEMPo com efeito aleatório para o nível dos animais

proc mixed noclprint covtest;

class animal dieta;

model teor $=$ tempo / solution $\mathrm{ddfm}=\mathrm{bw}$;

random intercept tempo / subject=animal type=un;

run;

. Programa para o modelo (20), considerando o TEMPO com efeito aleatório para o nível dos animais e a variável explanatória dieta desse nível

proc mixed noclprint covtest;

class animal dieta;

model $\mathrm{y}=$ tempo dieta /solution ddfm=bw;

random intercept tempo/subject=animal type=un;

run; 
Programa para o modelo (21), considerando o TEMPO com efeito aleatório para o nível dos animais e suas interações com a variável explanatória dieta desse nível

proc mixed noclprint covtest;

class animal dieta;

model teor $=$ tempo dieta tempo*dieta /solution ddfm=bw;

random intercept tempo/subject=animal type=un;

run; 


\section{APÊNDICE 2}

Resultados do MLwiN 
Resultados do MLwiN, para os dados do "PAI" com a variável pressão arterial sistólica sentado.

Resultado fornecido pelo modelo (16), considerando apenas os interceptos.

pass $_{\text {ocasião, idoso }} \sim \mathrm{N}(X B, \Omega)$

pass $_{\text {ocasião, idoso }}=\beta_{0 o c a s i a ̃ o, \text { idoso }}$ cons

$\beta_{\text {0ocasião, idoso }}=142,577(1,333)+u_{0 \text { idoso }}+e_{\text {0ocasião, idoso }}$

$\left[u_{\text {0idoso }}\right] \sim \mathrm{N}\left(0, \Omega_{u}\right): \Omega_{u}=[237,840(32,203)]$

$\left[e_{\text {Docasião, idoso }}\right] \sim \mathrm{N}\left(0, \Omega_{e}\right): \Omega_{e}=[315,303(14,178)]$

$-2 * \log$ likelihood(IGLS) $=10201,950(1154$ of 3652 cases in use) 
Resultado fornecido pelo modelo (17), considerando a variável explanatória IMCC fixa.

$$
\begin{aligned}
& \operatorname{pass}_{\text {ocasião, idoso }} \sim \mathrm{N}(X B, \Omega) \\
& \text { pass }_{\text {ocasião, idoso }}=\beta_{0 \text { ocasião, idoso }} \text { cons }+1,094(0,260) \text { imcc }_{\text {ocasiäo, idoso }} \\
& \beta_{\text {0ocasião, idoso }}=141,899(1,348)+u_{0 i d o s o}+e_{0 o c a s i a ̈ o, \text { idoso }} \\
& {\left[u_{\text {0idoso }}\right] \sim \mathrm{N}\left(0, \Omega_{u}\right): \Omega_{u}=[234,810(32,137)]} \\
& {\left[e_{\text {oocasião, idoso }}\right] \sim \mathrm{N}\left(0, \Omega_{e}\right): \Omega_{e}=[309,694(14,012)]} \\
& -2 \text { *loglikelihood(IGLS) }=10039,010(1138 \text { of } 3652 \text { cases in use })
\end{aligned}
$$


Resultado do MLwiN, para os dados do teor de proteína no leite.

Resultado fornecido pelo modelo (20), considerando as variáveis explanatórias TEMPO e DIETA de tremoço (lupins) e a mistura de cevada e tremoço (mixed) em relação à dieta de cevada.

$$
\begin{aligned}
& \text { teor }_{\text {obs, vaca }} \sim \mathrm{N}(X B, \Omega) \\
& \text { teor }_{\text {obs, vaca }}=\beta_{0 o b s, \text { vaca }} \text { cons }+\beta_{1 \text { vaca }} \text { tempo }_{\text {obs, vaca }}+-0,094(0,050) \text { mixed }_{\text {vaca }}+ \\
& -0,197(0,050) \text { lupins }_{\text {vaca }} \\
& \beta_{0 o b s, \text { vaca }}=3,617(0,045)+u_{0 v a c a}+e_{0 o b s, \text { vaca }} \\
& \beta_{\text {vaca }}=-0,013(0,003)+u_{1 \text { vaca }}
\end{aligned}
$$

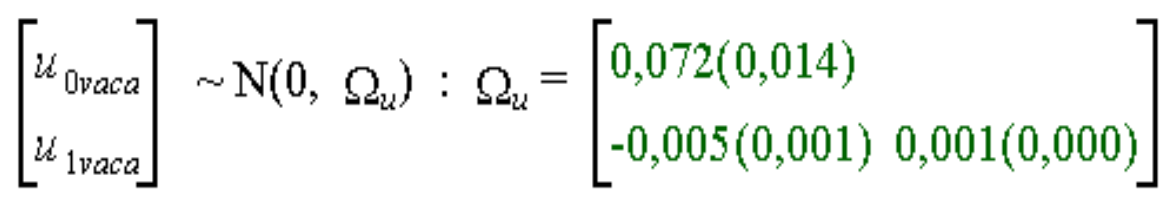

$$
\begin{aligned}
& {\left[e_{0 o b s, \text { vaca }}\right] \sim \mathrm{N}\left(0, \Omega_{e}\right): \Omega_{e}=[0,060(0,002)]}
\end{aligned}
$$

-2 *loglikelihood(IGLS) $=342,184(1337$ of 1501 cases in use $)$ 